\title{
Blood vessel segmentation algorithms - Review of methods, datasets and evaluation metrics
}

\author{
Sara Moccia, Elena De Momi, Sara El Hadji, and Leonardo S. Mattos
}

\begin{abstract}
Background: Blood vessel segmentation is a topic of high interest in medical image analysis since the analysis of vessels is crucial for diagnosis, treatment planning and execution, and evaluation of clinical outcomes in different fields, including laryngology, neurosurgery and ophthalmology. Automatic or semiautomatic vessel segmentation can support clinicians in performing these tasks. Different medical imaging techniques are currently used in clinical practice and an appropriate choice of the segmentation algorithm is mandatory to deal with the adopted imaging technique characteristics (e.g. resolution, noise and vessel contrast).
\end{abstract}

Objective: This paper aims at reviewing the most recent and innovative blood vessel segmentation algorithms. Among the algorithms and approaches considered, we deeply investigated the most novel blood vessel segmentation including machine learning, deformable model, and tracking-based approaches.

Method: This paper analyzes more than 100 articles focused on blood vessel segmentation methods. For each analyzed approach, summary tables are presented reporting imaging technique used, anatomical region and performance measures employed. Benefits and disadvantages of each method are highlighted.

Discussion: Despite the constant progress and efforts addressed in the field, several issues still need to be overcome. A relevant limitation consists in the segmentation of pathological vessels. Unfortunately, not consistent research effort has been addressed to this issue yet. Research is needed since some of the main assumptions made for healthy vessels (such as linearity and circular cross-section) do not hold in pathological tissues, which on the other hand require new vessel model formulations. Moreover, image intensity drops, noise and low contrast still represent an important obstacle for the achievement of a high-quality enhancement. This is particularly true for optical imaging, where the image quality is usually lower in terms of noise and contrast with respect to magnetic resonance and computer tomography angiography.

Conclusion: No single segmentation approach is suitable for all the different anatomical region or imaging modalities, thus the primary goal of this review was to provide an up to date source of information about the state of the art of the vessel segmentation algorithms so that the most suitable methods can be chosen according to the specific task.

Index Terms-Blood vessels, Medical imaging, Review, Segmentation.

S. Moccia is with the Department of Electronics, Information and Bioengineering, Politecnico di Milano, Milan, Italy and with the Department of Advanced Robotics, Istituto Italiano di Tecnologia, Genoa, Italy, e-mail: sara.moccia@polimi.it.

Sara El Hadji and Elena De Momi are with with the Department of Electronics, Information and Bioengineering, Politecnico di Milano, Milan, Italy.

Leonardo S. Mattos is with with the Department of Advanced Robotics, Istituto Italiano di Tecnologia, Genoa, Italy.

\section{INTRODUCTION}

Blood vessel analysis plays a fundamental role in different clinical fields, such as laryngology, oncology [1], ophthalmology [2], and neurosurgery [3]-[5] [6], both for diagnosis, treatment planning and execution, and for treatment outcome evaluation and follow up.

The importance of vessel analysis is supported by the constant introduction in clinical practice of new medical technologies aimed at enhancing the visualization of vessels, as endoscopy in Narrow Band Imaging (NBI) [7] and cone beam Computed Tomography (CT) 3D Digital Subtraction Angiography (DSA) [8]. At the same time, standard techniques, such as Magnetic Resonance Angiography (MRA) and Computed Tomography Angiography (CTA), are constantly improved to enhance vascular tree visualization [9], [10], [11].

Manual segmentation of blood vessels is an expensive procedure in terms of time and lacking intra- and interoperator repeatability and reproducibility. On the other hand, semi-automatic or automatic vessel segmentation methods require at least one expert clinician to segment or to evaluate the segmentation results obtained. In addition, support for the development and evaluation of such algorithms is still poor as publicly available image datasets with associated Gold Standard (GS) segmentation are currently limited to specific anatomical regions, such as retina [12]. However, automatic or semi-automatic blood vessel segmentation could assist clinicians and, therefore, are topics of great interest in medical research, as demonstrated by the high amount of papers annually published in this field. Indeed, an extensive literature already exists on vessel segmentation and in the past years different reviews on vessel segmentation algorithms have been published, such as [13], [14], [12], [15], [16], [17], [18] and [19]. However, due to the strong development in the field, updated reviews are required to analyze and summarize the actual state of the art.

This review aims at analyzing a wide spectrum of the most recent and innovative vessel segmentation techniques found in the literature, reporting on state of the art approaches based on machine learning (Sec. V), deformable model (Sec. VI) and tracking methods (Sec. VII]. Moreover, it reports on the most commonly adopted metrics for the evaluation of segmentation results (Sec. III) and identifies the available testing datasets (Sec. IV).

The goal of this review is to provide comprehensive information for the understanding of existing vessel segmentation algorithms by summarizing their advantages and limitations. Each segmentation approach is first analyzed in the general 


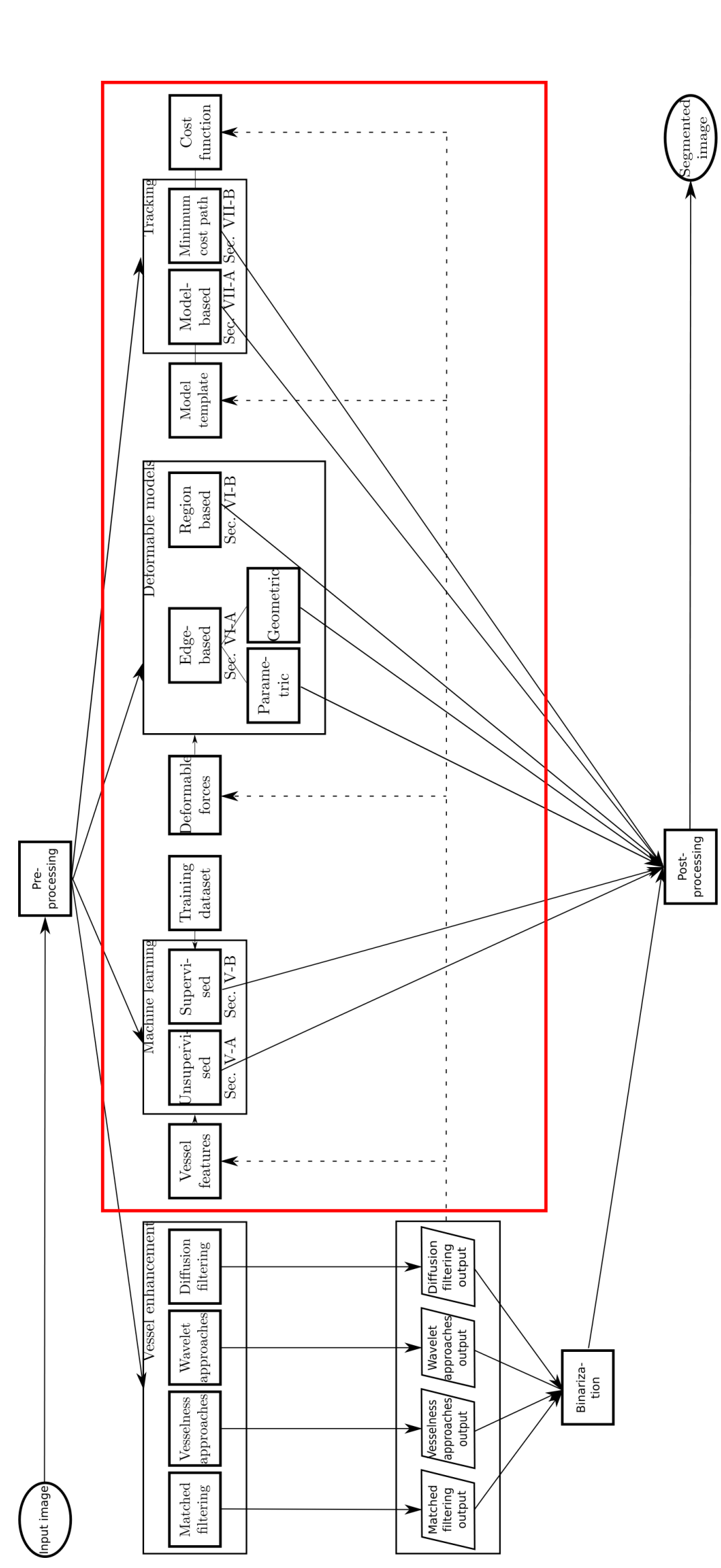

$\varangle$

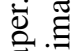

일

瓷

$\cdot 0$

के

:

흥

言

둥ㅎㅇ

ए

氜 获

च छ

语

कo है चี

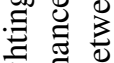

해뭉

.0요

ठ

ช.

这

ํㅝㄹ

造 훙

苋造

을.

.

呵

言苛

$\infty$ :

ऽ. ฮี

究

马्

긍

สิ

E

ن

용

过

3 ․

흐 츨

퐁

요

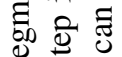

o 00.0

के है

$>$ \&े

- - 음

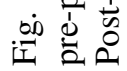


TABLE I: Vessel segmentation categorization. MRA: Magnetic Resonance Angiography, CT: Computed Tomography, CT:A Computed Tomography Angiography, CFP: Color Fundus Photography, OCT: Optical Coherence Tomography, US: Ultrasound, FA: Fluorescein Angiography, DSA: Digital Subtraction Angiography, 3DRA: 3D Rotational Angiography.

\begin{tabular}{|c|c|c|c|c|}
\hline Method & Year & Anatomical region & Imaging technique & Image processing method \\
\hline $\begin{array}{l}\text { Feng et al. 20 } \\
\text { Hassouna et al. } 21] \\
\text { Oliveira et al. } 22 \\
\text { Goceri et al. } 23 \\
\text { Bruyninckx et al. } 24 \\
\text { Bruyninckx et al. } 25 \\
\text { Asad et al. 26 } \\
\text { Mapayi et al. } 27 \\
\text { Sreejini et al. } 28 \\
\text { Cinsdikici et al. } 29 \\
\text { Al-Rawi et al. } 30\end{array}$ & $\begin{array}{l}2010 \\
2006 \\
2011 \\
2017 \\
2010 \\
2009 \\
2017 \\
2015 \\
2015 \\
2009 \\
2007\end{array}$ & $\begin{array}{l}\text { Brain } \\
\text { Brain } \\
\text { Liver } \\
\text { Liver } \\
\text { Liver } \\
\text { Lung } \\
\text { Retina } \\
\text { Retina } \\
\text { Retina } \\
\text { Retina } \\
\text { Retina }\end{array}$ & $\begin{array}{l}\text { MRA } \\
\text { MRA } \\
\text { CT } \\
\text { MRI } \\
\text { CT } \\
\text { CT } \\
\text { CFP } \\
\text { CFP } \\
\text { CFP } \\
\text { CFP } \\
\text { CFP }\end{array}$ & $\begin{array}{l}\text { Unsupervised machine learning } \\
\text { (Sec. V-A }\end{array}$ \\
\hline 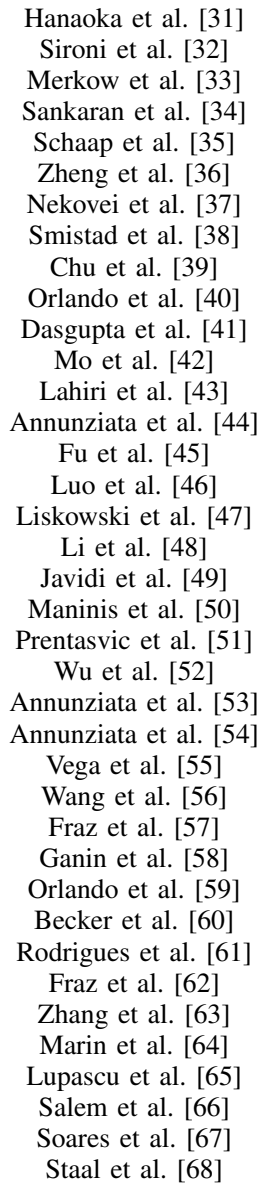 & $\begin{array}{l}2015 \\
2014 \\
2016 \\
2016 \\
2011 \\
2011 \\
1995 \\
2016 \\
2016 \\
2017 \\
2017 \\
2017 \\
2017 \\
2016 \\
2016 \\
2016 \\
2016 \\
2016 \\
2016 \\
2016 \\
2016 \\
2016 \\
2015 \\
2015 \\
2015 \\
2015 \\
2014 \\
2014 \\
2014 \\
2013 \\
2013 \\
2012 \\
2012 \\
2011 \\
2010 \\
2007 \\
2006 \\
2004\end{array}$ & 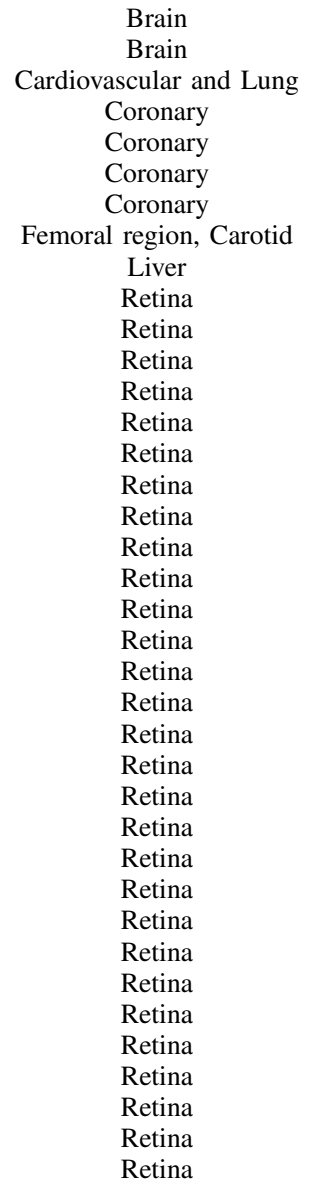 & $\begin{array}{c}\text { MRA } \\
\text { Microscopy } \\
\text { CT and MRI } \\
\text { CTA } \\
\text { CTA } \\
\text { CT } \\
\text { CT } \\
\text { US } \\
\text { X-ray fluoroscopic } \\
\text { CFP } \\
\text { CFP } \\
\text { CFP } \\
\text { CFP } \\
\text { Microscopy } \\
\text { CFP } \\
\text { CFP } \\
\text { CFP } \\
\text { CFP } \\
\text { CFP } \\
\text { CFP } \\
\text { CT } \\
\text { CFP } \\
\text { Microscopy } \\
\text { Microscopy } \\
\text { CFP } \\
\text { CFP } \\
\text { CFP } \\
\text { CFP } \\
\text { CFP } \\
\text { CFP } \\
\text { OCT } \\
\text { CFP } \\
\text { CFP } \\
\text { CFP } \\
\text { CFP } \\
\text { CFP } \\
\text { CFP } \\
\text { CFP }\end{array}$ & $\begin{array}{l}\text { Supervised machine learning } \\
\text { (Sec. V-B }\end{array}$ \\
\hline $\begin{array}{l}\text { Lee et al. } 69] \\
\text { Valencia et al. } 70 \\
\text { Law et al. } 71 \\
\text { Moreno et al. } 72] \\
\text { Wang et al. } 73 \\
\text { Cheng et al. } 74] \\
\text { Zhu et al. } \\
\text { Zhang et al. } 75 \\
\text { Patwardhan et al. } 177\end{array}$ & $\begin{array}{l}2015 \\
2007 \\
2009 \\
2013 \\
2012 \\
2015\end{array}$ & $\begin{array}{c}\text { Aorta \& mesenteric artery } \\
\text { Artery } \\
\text { Brain \& Coronary } \\
\text { Coronary } \\
\text { Coronary } \\
\text { Carotid,Coronary } \\
\text { Liver, \& Lung } \\
\text { Lung } \\
\text { Retina } \\
\end{array}$ & $\begin{array}{c}\text { CTA } \\
\text { MRA } \\
\text { MRA \& CTA } \\
\text { CTA } \\
\text { CTA }\end{array}$ & $\begin{array}{c}\text { Edge-based deformable models } \\
\text { (Sec.VI-A }\end{array}$ \\
\hline
\end{tabular}


TABLE I: Vessel segmentation categorization. MRA: Magnetic Resonance Angiography, CT: Computed Tomography, CT:A Computed Tomography Angiography, CFP: Color Fundus Photography, OCT: Optical Coherence Tomography, US: Ultrasound, FA: Fluorescein Angiography, DSA: Digital Subtraction Angiography, 3DRA: 3D Rotational Angiography.

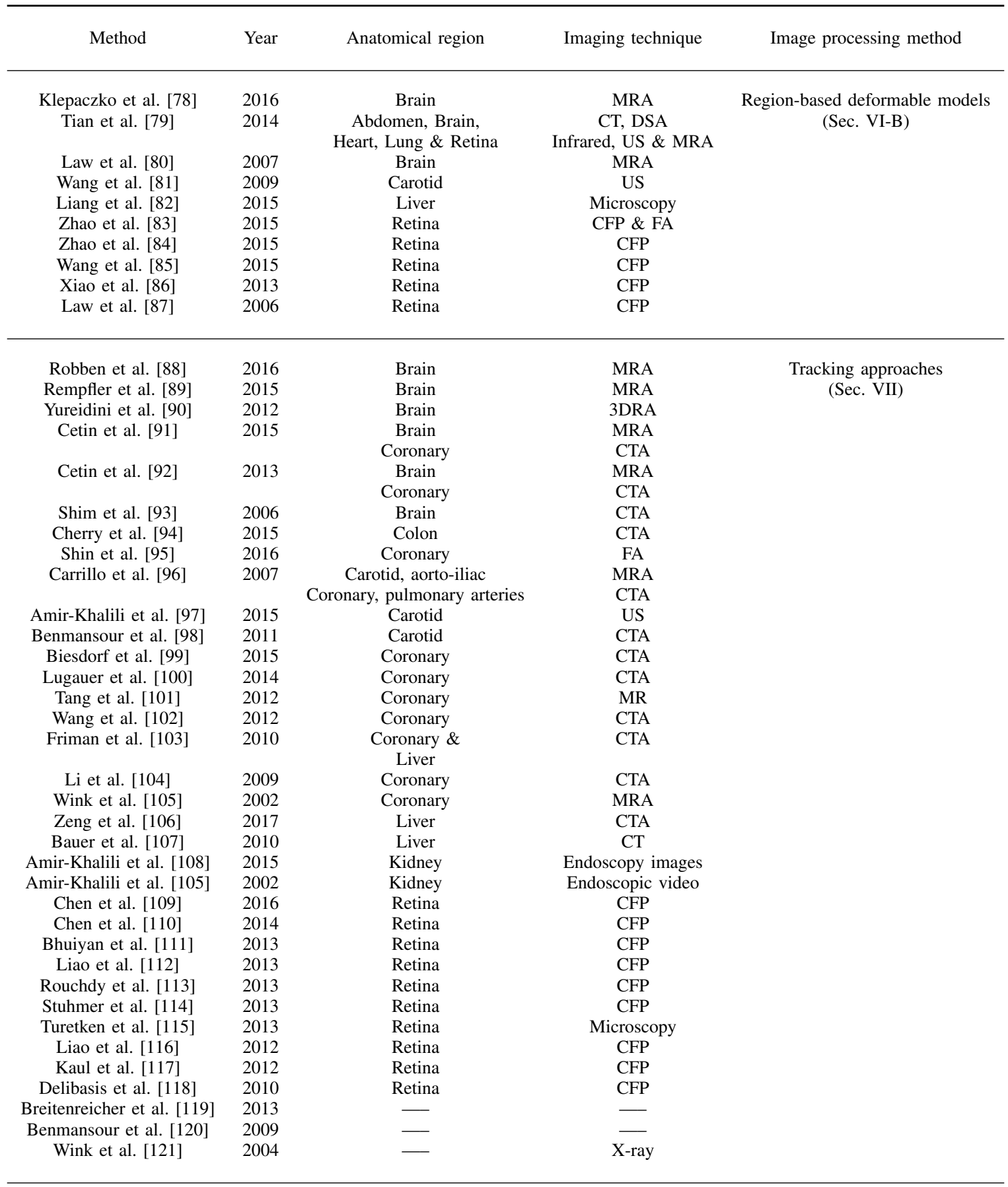


context of image segmentation and then in the specific context of vessel segmentation. For each segmentation category, papers are discussed, illustrating their benefits and potential disadvantages. In addition, summary tables reporting performance measures are presented for each category. The paper concludes with a discussion on future directions and open issues in the field of vessel segmentation.

A summary of the papers analyzed in this review considering year of publication, anatomical region and imaging technique is reported in Table I In addition, Fig. 1 highlights the categories of vessel segmentation algorithms analyzed in the following sections of this paper.

\section{Algorithm WORKFLOW}

As shown in Fig. 1, in vessel segmentation algorithms the input image first undergoes a pre-processing step, which typically concerns noise suppression, data normalization, contrast enhancement and conversion of color image to grayscale image. Since different imaging modalities produce images characterized by different resolution, noise and contrast, different pre-processing techniques have to be employed. An exhaustive review on pre-processing algorithms is presented in [122].

The core of the vessel segmentation workflow concerns the segmentation process, which can be classified in four different categories:

- Vessel enhancement

- Machine learning

- Deformable models

- Tracking

Through vessel enhancement approaches, the quality of vessel perception is improved, e.g. by increasing the vessel contrast with respect to background and other non-informative structures. A strong and established literature on vessel enhancement approaches already exists. Examples include matched filtering [123], vesselness-based approaches [124], Wavelet [67] and diffusion filtering [125]. Due to the extensive literature on the enhancement methods and the wideness of this subject, in this review we will not deal with it. A complete review on the topic can be found e.g. in [12].

The vessel enhancement can be followed by a thresholding step to directly obtain the vessel binary mask. Nonetheless, modern methods employ the enhanced vasculature as a preliminary step for more sophisticated segmentation algorithms. In particular, the enhanced vasculature can be used to extract features to be classified with machine learning algorithms (Sec. $\mid \mathrm{V}$ ), to define forces that constraint vessel model deformation for deformable model-based segmentation (Sec. VI), or to guide vascular tracking through enhanced vasculature intensity or gradient-based constraints (Sec. VII), as explained in depth in this review.

A post-processing step may also be employed, e.g. to reconnect vascular segments or remove too small segmented areas, which often correspond to image artifacts or noise.

\section{Evaluation Metrics}

Segmentation performance is commonly evaluated with respect to GS manual segmentation performed by an expert
TABLE II: Contingency table for vessel segmentation.

\begin{tabular}{|c|c|c|c|}
\cline { 3 - 4 } \multicolumn{2}{c|}{} & \multicolumn{2}{c|}{ Gold Standard segmentation } \\
\cline { 4 - 4 } \multicolumn{2}{c|}{} & Vessel & Non-vessel \\
\hline Algorithm & Vessel & $T P$ & $F P$ \\
segmentation & Non-vessel & $F N$ & $T N$ \\
\cline { 4 - 4 }
\end{tabular}

TABLE III: Performance measures for vessel segmentation algorithms.

\begin{tabular}{|c|c|}
\hline Index & Description \\
\hline Accuracy $(A c c)$ & $\frac{T P+T N}{n}$ \\
\hline Sensitivity $(\mathrm{Se})$ & $\frac{T P}{T P+F N}$ \\
\hline Specificity $(S p)$ & $\frac{T N}{T N+F P}$ \\
\hline False Positive rate ( $F P$ rate) & $1-S p$ \\
\hline Positive Predictive Value $(P P V)$ & $\frac{T P}{T P+F P}$ \\
\hline Negative Predictive Value $(N P V)$ & $\frac{T N}{T N+F N}$ \\
\hline$A U R O C$ & $\begin{array}{l}\text { Area Under the Receiver } \\
\text { Operating Characteristic curve }\end{array}$ \\
\hline $\begin{array}{c}\text { Matthews Correlation } \\
\text { Coefficient }(M C C)\end{array}$ & Eq. 1 \\
\hline Cohen's $\kappa$ coefficient $(\kappa)$ & Eq. 2 \\
\hline Dice Similarity Coefficient ( $D S C$ ) & Eq. 3 \\
\hline Hausdorff Distance $H D$ & Eq. 4 \\
\hline Connectivity & Eq. 5 \\
\hline Area & Eq. 6 \\
\hline Length & Eq. 7 \\
\hline Overlap $(O V)$ & Eq. 8 \\
\hline Overlap until first error $(O F)$ & Eq. 9 \\
\hline $\begin{array}{c}\text { Overlap with clinically relevant } \\
\text { part of the vessel }(O T)\end{array}$ & Eq. 10 \\
\hline
\end{tabular}

clinician. To attenuate intra-subject variability when performing the manual segmentation, and obtain a truthful GS, a combination of segmentations by multiple experts is usually employed. Different strategies have been proposed to combine the segmentations: for example, a voting rule, often used in practice, selects as GS all voxels where the majority of experts agree the structure to be segmented is present [126]. However, such approach does not allow for incorporating a priori information of the structure being segmented or estimating the presence of an imperfect or limited reference standard.

To solve this issue, the Simultaneous Truth And Performance Level Estimation (STAPLE) has been introduced in [127]. The approach takes a collection of segmentations and computes simultaneously a probabilistic estimate of the true segmentation and a measure of the performance level represented by each segmentation using an ExpectationMaximization (EM) algorithm.

When evaluating the performance of segmentation algorithms with respect to GS, a contingency table (Table III) with True Positive $(T P)$, True Negative $(T N)$, False Negative $(F N)$, and False Positive $(F P)$ is commonly used, where positive and negative refer to pixels belonging to vessels and background as in accord with the GS segmentation, respectively.

Segmentation performance measures are summarized in Table III Accuracy $(A c c)$, Sensitivity $(S e)$, and Specificity $(S p)$ 
are the most frequently adopted measures, where $A c c$ is the proportion of true results, both $T P$ and $T N$, among the total number of examined cases $(n) . S e$, also referred as $T P$ rate, measures the proportion of positives, both $T P$ and $F N$, that are correctly identified. $S p$ measures the proportion of negatives, both $T N$ and $F P$, that are correctly identified. Although a high $S e$ reflects the desirable algorithm inclination to detect vessels, a high $S e$ with low $S p$ indicates that the segmentation includes many pixels that do not belong to vessels, i.e. high FP. Consequently, an algorithm that provides high $S e$ and low $S p$ is acceptable if the post-processing step is able to remove possible $F P$.

Despite the fact that $A c c, S e$ and $S p$ are the most frequently adopted performance metrics, other derived metrics are also often employed. Examples include $F P$ rate, which is equal to $1-S p$, Positive Predictive Value $(P P V)$, which is the proportion of $T P$ among $T P+F P$, and Negative Predictive Value $(N P V)$, which is the ratio between $T N$ and $T N+$ $F N$. PPV gives an estimation of how likely it is that a pixel belongs to a vessel given that the algorithm classifies it as positive. NPV corresponds to the likelihood that a pixel does not belong to a vessel, given that the algorithm classifies it as negative.

Receiver Operating Characteristic $(R O C)$ curve, which illustrates the performance of a binary classifier system as its discrimination threshold is varied, is also often reported. The area under the $R O C(A U R O C)$ is used as a metric, indicating the probability that a classifier will rank a randomly chosen positive instance higher than a randomly chosen negative one. $A U R O C$ assumes value 1 for a perfect classifier. Different algorithms for the $A U R O C$ estimation are reported in the literature [128]. Precision-recall curve can be used, too. Precision corresponds to $P P V$, while recall to $S e$. The precisionrecall curve compares $T P$ with $F N$ and $F P$, excluding $T N$, which is less relevant for the vessel segmentation performance evaluation since the proportion of $T P$ (vessels) and $T N$ (background) is highly skewed. Also in this case, the area under the precision-recall curve $(A U P R C)$ can be exploited.

Another metric that can be used is the Matthews Correlation Coefficient $(M C C)$ [129]:

$$
\begin{aligned}
& M C C=(T P * T N)-(F P * T N) \\
& \sqrt{((T P+F P)(T P+F N)(T N+F P)(T N+F N)}
\end{aligned}
$$

Some authors also report the Cohen's $\kappa$ coefficient [130], which is a measures of inter-rater agreement:

$$
\kappa=\frac{A c c-p_{e}}{1-p_{e}}
$$

where $p_{e}$ is the hypothetical probability of chance agreement, equal to the probability of GS to generate positives times the probability of the algorithm to generate positives. Cohen's $\kappa$ coefficient is considered a robust metric since it takes into account also the agreement between algorithm and GS occurring by chance.

Spatial overlapping indexes can be used, too. The most used is the Dice Similarity Coefficient $(D S C)$ [131], which is computed as the ratio of the number of elements (card) in the intersection of two clusters $A$ and $B$ by the mean label image, where $A$ and $B$ indicate the segmented vessels and its corresponding GS, respectively:

$$
D S C=\frac{\operatorname{card}(A \cap B)}{\frac{\operatorname{card}(A)+\operatorname{card}(B)}{2}}=\frac{2 T P}{F P+F N+2 T P}
$$

DSC is also known as $F_{1}$ score.

Hausdorff distance is another overlapping index, which measures how far the GS segmentation and the segmented image are from each other:

$$
\begin{aligned}
& H D= \\
& \max \left(\sup _{a \in A} i n f_{b \in B} d(a, b), \sup _{b \in B} i n f_{a \in A} d(a, b)\right)
\end{aligned}
$$

where sup represents the supremum, inf the infimum and $d$ is a chosen metric, e.g. absolute value distance.

All metrics described above are based on the pixel-to-pixel comparison between the segmented image and the GS, without considering that vessel pixels are part of a connected vascular structure with specific features, such as area and length. For this reason, the use of three additional metric functions is suggested in [132]:

$$
\begin{gathered}
\text { Connectivity }=1-\min \left(1, \frac{|\operatorname{card}(A)-\operatorname{card}(B)|}{\operatorname{card}(A)}\right) \\
\text { Area }=\frac{\left.\operatorname{card}\left(\left(\delta_{r_{1}}(A) \cap B\right) \cup\left(\delta_{r_{1}}(B) \cap A\right)\right)\right)}{\operatorname{card}(A \cup B)} \\
\text { Length }=\frac{\left.\operatorname{card}\left(\psi(A) \cap \delta_{r_{2}}(B)\right) \cup\left(\delta_{r_{2}}(A) \cap \psi(B)\right)\right)}{\operatorname{card}(\psi(A) \cup \psi(B))}
\end{gathered}
$$

where $\delta_{r_{1}}$ and $\delta_{r_{2}}$ are morphological dilatations obtained using a disc of radius $r_{1}$ and $r_{2}$, respectively, and $\psi$ is a homotopic skeletonization [133]. The Connectivity term penalizes fragmented segmentation. The Area factor measures the degree of overlapping between $A$ and $B$, being less sensitive to slight differences between the segmentation and the GS if compared to the $D S C$, due to the introduced dilatation. The Length factor evaluates the consistency between the segmented and the GS vessel length.

Another class of evaluation metrics proposed in [134] aims at quantifying the performance of segmentation algorithms in terms of point-to-point correspondence between the GS vessel centerline and the computed centerline. The point correspondence is commonly computed with the mean shift algorithm [135] and three different centerline overlap measures are derived. The overlap $(O V)$ measures the ability to track the overall vessel annotated by the observers and it is defined as follows:

$$
O V=\frac{T P M_{o v}+T P R_{o v}}{T P M_{o v}+T P R_{o v}+F N_{o v}+F P_{o v}}
$$

where $T P R_{o v}$ refers to points of the GS centerline whose distance to the correspondent points on the evaluated centerline is less than the local vessel radius. Points for which this distance is higher than the radius are marked as $F N_{o v}$. Points on the evaluated centerline are marked as $T P M_{o v}$ if there is at least one point on the GS at a distance less than the radius, otherwise they are marked as $F P_{o v}$. 
TABLE IV: Publicly available databases with associated Gold Standard segmentation. CFP: Color Fundus Photography, CTA: Computed Tomography Angiography, CT: Computed Tomography, FA: Fluorescein Angiography.

\begin{tabular}{|c|c|c|}
\hline Name & $\begin{array}{l}\text { Anatomical } \\
\text { region }\end{array}$ & $\begin{array}{c}\text { Number of } \\
\text { images/volumes }\end{array}$ \\
\hline STARE 136 . & Retina & $20 \mathrm{CFP}$ \\
\hline DRIVE 68] & & $40 \mathrm{CFP}$ \\
\hline ARIA [137] & & 143 CFP \\
\hline CHASE [138] & & 28 CFP \\
\hline HRF 139] & & 45 CFP \\
\hline IMAGERET [140] & & $219 \mathrm{CFP}$ \\
\hline $\begin{array}{c}\text { MESSIDOR } \\
\text { (http://messidor.crihan.fr) }\end{array}$ & & $1200 \mathrm{CFP}$ \\
\hline REVIEW 141] & & 16 CFP \\
\hline $\mathrm{ROC}[142]$ & & $100 \mathrm{CFP}$ \\
\hline $\begin{array}{c}\text { VICAVR } \\
\text { ( http://www.varpa.es) }\end{array}$ & & $58 \mathrm{CFP}$ \\
\hline $\begin{array}{l}\text { VAMPIRE } \\
\text { (http://vampire.computing. } \\
\text { dundee.ac.uk) }\end{array}$ & & $8 \mathrm{FA}$ \\
\hline $\begin{array}{l}\text { CASDQEF } 143 \\
\text { ROTTERDAM } 1144]\end{array}$ & Coronary & $\begin{array}{l}48 \text { CTA } \\
20 \text { CTA }\end{array}$ \\
\hline VESSEL12 145] & Lung & $20 \mathrm{CT}$ \\
\hline $\begin{array}{c}\text { 3D-IRCADb } \\
\text { (http://www.ircad.fr/research/3dircadb/) }\end{array}$ & Liver & $22 \mathrm{CT}$ \\
\hline $\begin{array}{c}\text { OSMSC } \\
\text { (http://www.vascularmodel.com) }\end{array}$ & $\begin{array}{l}\text { Cardiovascular and } \\
\text { Lung }\end{array}$ & $93 \mathrm{MRA}$ and $\mathrm{CT}$ \\
\hline Vascular Synthesizer 146] & 3D Synthetic data & 120 \\
\hline
\end{tabular}

The overlap until first error $(O F)$ is the ratio of the number of $T P R_{o v}$ before the first error $\left(T P R_{o f}\right)$ and the overall number of reference points $(N R)$ :

$$
O F=\frac{T P R_{o f}}{N R}
$$

The first error refers to the first point of the GS centerline that is at a distance higher than the radius from the correspondent point on the evaluated centerline.

Overlap with the clinically relevant part of the vessel $(O T)$ provides an estimation of the ability of the method to segment vessel segments that are considered clinically relevant, e.g. have a diameter equal or larger then $1.5 \mathrm{~mm}$. In this case, the point $\left(\mathbf{p}_{\text {end }}\right)$ closest to the end of the reference GS with a radius larger than or equal to $0.75 \mathrm{~mm}$ is determined and $O T$ is computed as:

$$
O T=\frac{T P M_{o t}+T P R_{o t}}{T P M_{o t}+T P R_{o t}+F N_{o t}+F P_{o t}}
$$

where $T P M_{o t}, T P R_{o t}, F N_{o t}, F P_{o t}$ are computed as $T P M_{o v}, T P R_{o v}, F N_{o v}, F P_{o v}$ but considering only points between $p_{e n d}$ and the beginning of the GS centerline.

\section{Evaluation DATASETS}

Phantoms presenting meaningful features of interest with respect to the vascular tree (e.g. intensity profile, thickness, tor-

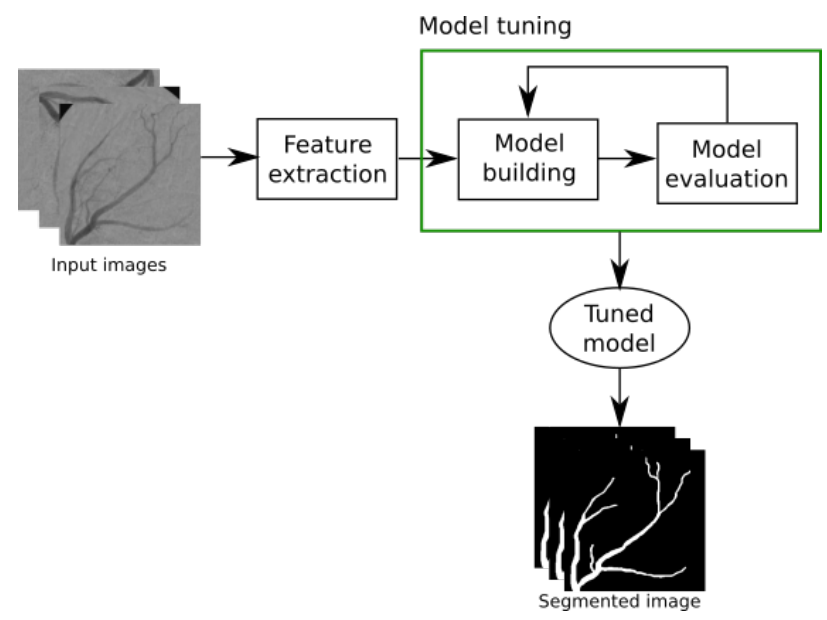

Fig. 2: Unsupervised learning approaches build segmentation models based on unlabeled image features, such as local intensity and gradient. During model tuning, the goodness of the model is evaluated and the model is tuned according to a minimization function that aims at finding the best separation between the vascular and background classes. Usually, such function is defined upon metrics such as Euclidean or probabilistic distance.

tuosity) are often considered for the evaluation of segmentation algorithms. Phantoms are easy to control and modify with the goal of understanding how and to which degree the algorithm performance depends on parameter settings. Moreover, by providing a GS, phantoms allow simple algorithm validation and training, which is not always an easy task for real clinical images since the correspondent GS may not be available [146]. Considering the benefit of using phantoms, methods to develop realistic digital phantoms have been presented, e.g in [78] for intracranial arterial tree on time-of-flight MRI.

Although phantoms have an important role in quantifying algorithm performance, they do not always fully reflect clinical images, e.g. due to the high inter-patient variability. To overcome this issue, a number of publicly available databases with associated GS has been published in the last few years. Publicly available databases encourage consistent and fair comparison of vessel segmentation algorithms. However, this positive trend still concerns only a few anatomical regions. A list of publicly available databases is presented in Tab. IV

\section{MACHINE LEARNING}

There are two main classes of machine learning approaches: unsupervised and supervised. The former finds a model able to describe hidden arrangement of input image-derived features, without any prior knowledge or supervision, while the latter learns a data model from a set of already labeled features, as explained in Sec. V-A and V-B respectively. Since unsupervised learning does not require GS segmentation, it is useful for cases where publicly available GS datasets are not available as well as for exploratory data analysis. On the other hand, supervised learning requires GS segmentation to train the learning model. The training computational cost varies depending on the adopted supervised learning approach. 
However, during testing phases, the computational cost is usually negligible.

\section{A. Unsupervised}

Unsupervised learning approaches represent particular features on the base of the statistical distribution of the overall input data. The absence of available GS for supervised training justifies the employment of such methods, at the cost of segmentation performance usually less satisfying with respect to the supervised approach ones. A typical unsupervised learning algorithm workflow is shown in Fig. 2 .

In [21], stochastic modeling is used to segment cerebrovascular structures from time of flight MRA. The pixel intensity histogram is described by two major classes: vessel and background. Background class is approximated by two Gaussians and one Rayleigh distribution, while the vessel class is approximated by one Gaussian. EM algorithm [147] is employed to automatically estimate the Gaussians and Rayleigh distribution parameters. Spatial constraints are included through Markov Random Field (MRF) modeling [148], privileging connected sets of data. Thus, MRF are particularly useful when high noise level is present in the images.

In [23], k-means clustering is used for rough liver vessel segmentation. Further iterative refinement steps based on morphological operations are applied to refine the segmentation. This method relies on automatic k-means and morphological operator parameter selection. Thus, the algorithm can adapt to different pixel intensity distributions in the image.

In [22], liver vessel segmentation is performed with regiongrowing in CT images. A pixel is incorporated in the growing region if its intensity falls in a predefined range. The range extrema are defined by approximating the image histogram with three Gaussians, through Gaussian Mixture Model (GMM) [149]. This method is relevant for several imaging techniques, as far as the pixel intensity distribution is nearly Gaussian shaped. Portal and hepatic veins are subsequently separated according to geometric features as dimension and connectivity. In [20], a similar approach is used to segment the brain vascular pattern. Maximum Intensity Projection (MIP) version of the CT is used to enhance the vascular structures and only two classes are considered for vessel and background in the histogram approximation, resulting in a lower GMM computational cost.

Fuzzy C-means segmentation of retinal blood vessels is employed in [27]. To face non-uniform illumination and contrast, phase-congruency $[150]$ is first performed, which preserves features with in-phase frequency components, such as edges, while suppressing the others. Consequently, accurate segmentation can be performed also in presence of intensity drops and varying illumination levels in the image.

In [28], Particle Swarm Optimization (PSO) is used to segment retinal vessels. PSO is used to iteratively find the optimal matched filter (MF) [123] parameters. The MF locally exploits the correlation between local image areas and filter kernel that reproduces the blood vessel architecture in terms of width and orientation. During the PSO iterative process, the $A U R O C$ of the $\mathrm{MF}$ response is used as fitness function

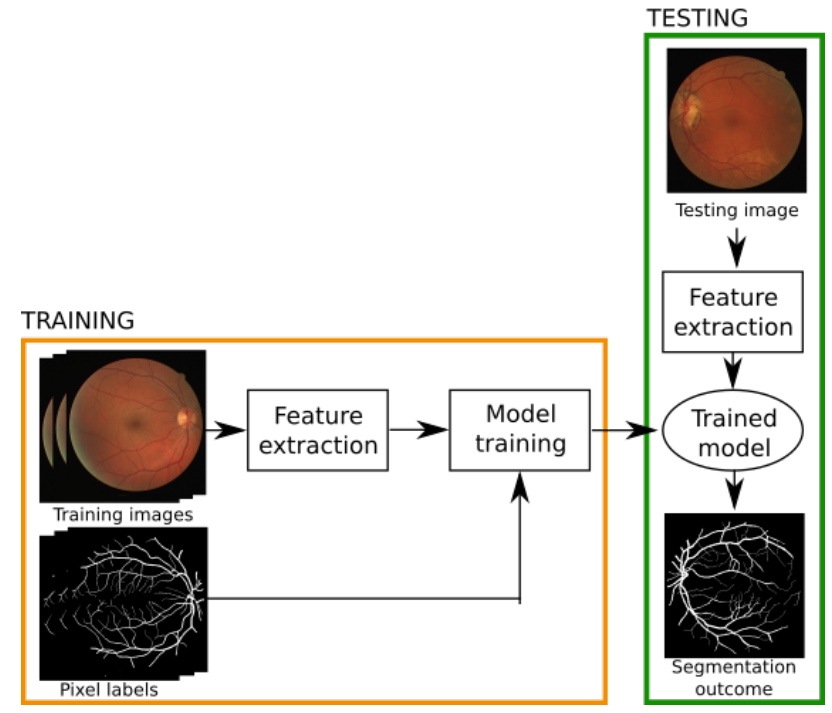

Fig. 3: Supervised approach workflow. During the training phase, image features (e.g. intensity, gradient, color) are extracted from the training images. A machine learning model is trained with such features and the corresponding labels, taken from the gold standard segmentation. Once the model is trained, it can be applied to a new, unseen, testing image to obtain the vessel segmentation.

for the PSO. Two MFs are employed to separately enhance small and thick vessels. Similarly, optimal MF parameters are retrieved using genetic algorithm in [30]. However, PSO has the shortcoming of easily falling into local optima, influencing the segmentation performance.

In [25], [24] ant colony optimization, a population-based metaheuristic used to find approximate solutions to optimization problems, is used to segment both lung and liver vessels. The method connects vessel bifurcation by cost path algorithm and uses the ant colony optimization method to retrieve the optimal vessel tree between all possible paths. A similar approach is exploited in [26] to segment retinal vessels. Ant colony segmentation results are combined with MF ones in [29] to improve the retinal vessel segmentation accuracy.

A summary of the analyzed Unsupervised approaches is presented in Table V

\section{B. Supervised}

Supervised learning for vessel segmentation infers a rule from labeled training couples, one for each pixel, which consist of an input vector of features (such as pixel intensity, MF response, etc.), and an output value, which states whether the pixel belongs to a vessel or not according to a GS. The workflow of a typical supervised approach is shown in Fig. 3

From the first attempts of using machine learning for vessel segmentation (including [37], [67], [68]), several algorithms have been published following a continuous progress of research on the topic. So far, supervised learning has been mainly applied to retinal images, since different labeled databases for training are publicly available (Tab IV]. 
TABLE V: Summary of unsupervised blood vessel segmentation algorithms (for performance indexes refer to Tab. III). CT: Computed Tomography, MRA: Magnetic Resonance Angiography.

\begin{tabular}{|c|c|c|c|}
\hline Method & Testing dataset & Synthetic data & Segmentation performance measure \\
\hline Feng et al. |20] & $136 \mathrm{MR}$ slices & No & Visual \\
\hline Hassouna et al. [21] & MRA & Yes & Visual \\
\hline Goceri et al. 23] & $14 \mathrm{MRI}$ & No & $D S C, H D$ \\
\hline Bruyninckx et al. 24 & $\begin{array}{c}5 \text { CT images (3D-IRCADb-01) } \\
\text { (http://www.ircad.fr/research/3dircadb/) }\end{array}$ & No & $D S C$ \\
\hline Bruyninckx et al. 25] & $1 \mathrm{CT}$ volume & No & Euclidean distance \\
\hline Oliveira et al. 22] & $15 \mathrm{CT}$ volumes & No & Visual \\
\hline Al-Rawi et al. 30 & 20 images (DRIVE database $[68 \mid$ ) & No & $A U R O C=0.96$ \\
\hline Asad et al. 26] & 20 images (DRIVE database $[68]$ ) & No & $S e=0.75$ \\
\hline Cinsdikici [29] & 20 images (DRIVE database $[68]$ ) & No & $A U R O C=0.94$ \\
\hline Mapayi et al. 27 & $\begin{array}{l}20 \text { images (STARE database }|136| \text { ) } \\
20 \text { images (DRIVE database }|68| \text { ) }\end{array}$ & No & $\begin{array}{l}A c c=0.93 \\
A c c=0.94\end{array}$ \\
\hline Sreejini et al. 28 & $\begin{array}{l}20 \text { images (STARE [136|) } \\
20 \text { images (DRIVE database }|68| \text { ) }\end{array}$ & No & $\begin{array}{l}A c c=0.95, S e=0.72, S p=0.97 \\
A c c=0.96, S e=0.71, S p=0.99\end{array}$ \\
\hline
\end{tabular}

In [61], retinal vasculature is segmented from Optical Coherence Tomography (OCT) images. A set of 2D fundus reference images are computed from the 3D OCT volume and used as input to a Support Vector Machine (SVM) with the Gaussian kernel. The effectiveness of the method depends on the choice of the selected SVM kernel as well as on the tuning of its parameters. This approach is able to segment both healthy and pathological retinal vessels. As a result, the study of disease progression is one of the major fields of application of this method.

In [31] a geometrical feature set is defined to classify cerebral vessel morphology in MRA. The features are specifically chosen to account also for morphologically abnormal lesions, making the algorithm suitable for segmented pathological structures. Vessel segmentation is obtained via region-growing and the binary vessel mask is approximated by a graph, whose nodes belong to the vessel tree. For each node, the 3D histogram of shortest path lengths between the considered node and the others is computed and used as feature vector. SVM is used to classify vascular tree morphology in healthy or pathological condition.

Fully-Connected Conditional Random Field (FCCRF) is used in [59] and [40] to segment retinal vessels in color fundus photography. FCCRF maps the image into a fully connected graph structure, in which every pixel (graph node) is influenced by the others. Each pixel is represented by a set of features extracted through vessel enhancement approaches, such as gradient magnitude, and MF response. Structured SVM [153] are employed to learn the FCCRF parameters. The fully-connected framework leads to a more robust segmentation with respect to the classification performed considering each vessel pixel as an isolated point, or as influenced by a restricted neighborhood (as for traditional CRF).

RAdius-based Clustering ALgorithm (RACAL), introduced in [154], is used in [66] to segment retinal vasculature in color fundus photography. RACAL is used to cluster pixels, through a distance-based principle, in the feature space built considering green channel intensity, gradient magnitude and maximum image Hessian $(H)$ eigenvalue. The assignment of each cluster to either the vessel or background class is made according to a training procedure, employing as GS a thresholded version of the vesselness:

$$
V_{\text {Salem }}=\frac{\max _{\sigma}\left(\lambda_{2}(\sigma)\right)}{\phi_{s t d}\left(\mathbf{e}_{\mathbf{1}}\right)}
$$

where $\mathbf{e}_{\mathbf{1}}$ is the $H$ eigenvector associated to the smallest eigenvalue, $\lambda_{2}$ is the biggest $H$ eigenvalue, and $\phi_{\text {std }}$ the standard deviation of the orientation of $\mathbf{e}_{\mathbf{1}}$ computed with different scale value $(\sigma)$. $\mathbf{e}_{\mathbf{1}}$ inclination is constant for longitudinal vessels thus providing a high vesselness value. Sparse coding is used in [49], [63] for retinal vessel enhancement and segmentation. Sparse coding approximates the image intensity by a sparse linear combination of items from an overcomplete dictionary built from training images patches.

In [36], Probabilistic Boosting Tree (PBT), is used to segment coronary arteries in CT images. Geometric features, which describe the position of an image voxel in a heartoriented coordinate system, are used. In addition, image steerable features are included in the boosting phase, which takes into account image intensity and gradient information. A similar approach is exploited in [57] to segment retinal vessels. In this case, the feature vector consists of Gabor Filter (GF) [67] and Gaussian filter outputs. A similar approach is used in [62]. 
TABLE VI: Summary of supervised blood vessel segmentation algorithms (for performance indexes refer to Tab. III). CT: Computed Tomography, CTA: Computer Tomography Angiography, MRA: Magnetic Resonance Angiography, US: Ultrasound.

\begin{tabular}{|c|c|c|c|}
\hline Method & Testing dataset & Synthetic data & Segmentation performance measure \\
\hline Hanaoka et al. 31] & 300 MRA volumes & No & ROC curve \\
\hline Annunziata et al. 44 & 140 Microscopy images & No & Precision-recall curve \\
\hline Annunziata et al. 53 & $\begin{array}{l}50 \text { images (IVCM database }[151 \mid) \\
1 \text { image (BF2D database }|152|) \\
1 \text { image (VC6 database }|152|)\end{array}$ & No & Visual, Precision-recall curve \\
\hline Annunziata et al. |54 & $\begin{array}{l}50 \text { images (IVCM database }[151]) \\
1 \text { image (BF2D database }[152]) \\
1 \text { image (VC6 database }[152])\end{array}$ & No & $\begin{array}{l}A U P R C=0.91 \\
A U P R C=0.81 \\
A U P R C=0.84\end{array}$ \\
\hline Sironi et al. 32 & 13 Microscopy images & No & Precision-recall curve \\
\hline Sankaran et al. 34 & 4697 CTA sections & No & $\mathrm{AUC}=0.90, \mathrm{Se}=0.90, \mathrm{Sp}=0.85$ \\
\hline Schaap et al. 35 & 24 CTA (ROTTERDAM database [144 & No & $\begin{array}{c}O V=0.97, O F=0.72 \\
O T=0.97\end{array}$ \\
\hline Merkow et al. 33 & $93 \mathrm{MRI}$ and CT volumes (OSMSC database) & No & Precision-recall curve, $P P V=0.42$ \\
\hline Nekovei et al. 37 & $1 \mathrm{CT}$ volume & No & $A c c=0.92$ \\
\hline Prentasvic et al. 51 & $80 \mathrm{CT}$ volumes & No & $A c c=0.83$, ROC curve \\
\hline Zheng et al. [36] & $14 \mathrm{CT}$ volumes & No & FPrate, $S e$ \\
\hline Chu et al. 39 & $6 \mathrm{X}$-ray fluoroscopic images & No & Visual \\
\hline Rodrigues et al. 61 & 48 OCT volumes & No & $\begin{array}{c}\text { Acc }=0.98, S e=0.81, S p=0.99 \\
\text { Connectivity }=0.98, \text { Area }=0.91 \\
\text { Length }=0.88, \kappa=0.85\end{array}$ \\
\hline Smistad et al. 38 & $\begin{array}{l}15 \text { US Femoral images } \\
2 \text { US Carotid images }\end{array}$ & No & $\begin{array}{l}A c c=0.94 \\
A c c=0.96\end{array}$ \\
\hline Fraz et al. 57 & 28 images (CHASE [138]) & No & $S e=0.74, S p=0.98, A c c=0.96$ \\
\hline Wu et al. [52] & 20 images (DRIVE database [68) & No & $A U R O C=0.97$ \\
\hline Ganin et al. $\mid 58$ & 20 images (DRIVE database [68] & No & $A U R O C=0.89$ \\
\hline Orlando et al. [59] & 20 images (DRIVE database 68 ) & No & $S e=0.78, S p=0.97$ \\
\hline Becker et al. [60] & 20 images (DRIVE database [68] & No & Precision-recall curve \\
\hline Lahiri et al. 43 & 20 images (DRIVE database [68]) & No & $A U R O C=0.96$ \\
\hline Luo et al. 46] & 20 images (DRIVE database 68]) & No & $A c c=0.95, S e=0.75$ \\
\hline Dasgupta et al. 41 & 20 images (DRIVE database [68) & No & $A c c=0.95, S e=0.75$ \\
\hline Lupascu et al. 65 & 20 images (DRIVE database [68]) & No & $\begin{array}{c}S e=0.67, S p=0.99 \\
A c c=0.96, A U R O C=0.96\end{array}$ \\
\hline Salem et al. 66 & 20 images (STARE database [136) & No & $S e=0.81$, FPrate $=0.04$ \\
\hline Javidi et al. [49] & $\begin{array}{l}20 \text { images (DRIVE database } \mid \sqrt{68}) \\
20 \text { images (STARE database }|\overline{136}|)\end{array}$ & No & $\begin{array}{c}S e=0.72, S p=0.97, A c c=0.94 \\
S e=0.780, S p=0.96, A c c=0.95\end{array}$ \\
\hline Maninis et al. |50] & $\begin{array}{l}20 \text { images (DRIVE database }[68 \mid) \\
10 \text { images (STARE database }[\overline{136} \mid)\end{array}$ & No & $\begin{array}{l}D S C=0.82, \text { Precision-recall curve } \\
D S C=0.83, \text { Precision-recall curve }\end{array}$ \\
\hline Vega et al. [55] & 20 images (DRIVE database [68]) & & $\begin{array}{c}A c c=0.94, S e=0.74 \\
S p=0.96, D S C=0.69, M C C=0.66\end{array}$ \\
\hline Wang et al. 56 & 20 images (STARE database [136]) & No & $\begin{array}{c}A c c=0.94, S e=0.70 \\
S p=0.97, D S C=0.66, M C C=0.64 \\
\mathrm{Se}=0.74, S p=0.98 \\
\text { Acc }=0.95, A U R O C=0.97\end{array}$ \\
\hline
\end{tabular}


TABLE VI: Summary of supervised blood vessel segmentation algorithms (for performance indexes refer to Tab. III. OCT: Optical Coherence Tomography.

\begin{tabular}{|c|c|c|c|}
\hline Method & Testing dataset & Synthetic data & Segmentation performance measure \\
\hline Zhang et al. 63. & $\begin{array}{l}20 \text { images (STARE database }[136] \text { ) } \\
20 \text { images (DRIVE database }[\overline{68} \text { ) } \\
20 \text { images (STARE database }[\overline{136} \text { ) }\end{array}$ & No & $\begin{array}{c}\mathrm{Se}=0.75, S p=0.98 \\
\mathrm{Acc}=0.95, A U R O C=0.98 \\
F P R=0.01, T P R=0.58, P P V=0.84 \\
F P R=0.01, T P R=0.74, P P V=0.74\end{array}$ \\
\hline Marin et al. [64] & $\begin{array}{l}40 \text { images (DRIVE database [68]) } \\
20 \text { images (STARE database [136]) }\end{array}$ & No & $\begin{array}{c}S e=0.71, S p=0.98, A c c=0.94 \\
P P V=0.84, N P V=0.96, A U R O C=0.97 \\
S e=0.69, S p=0.98, A c c=0.95 \\
P P V=0.82, N P V=0.97, A U R O C=0.98\end{array}$ \\
\hline Soares et al. 67] & $\begin{array}{l}19 \text { images (STARE database }|136| \text { ) } \\
20 \text { images (DRIVE database }|68| \text { ) }\end{array}$ & $\begin{array}{l}\text { No } \\
\text { No }\end{array}$ & $\begin{array}{l}A U R O C=0.97, A c c=0.95 \\
A U R O C=0.96, A c c=0.95\end{array}$ \\
\hline Staal et al. 68 & $\begin{array}{l}19 \text { images (STARE database }|136| \text { ) } \\
20 \text { images (DRIVE database }|68| \text { ) }\end{array}$ & $\begin{array}{l}\text { No } \\
\text { No }\end{array}$ & $\begin{array}{c}A c c=0.93 \\
A U R O C=0.95, A c c=0.94\end{array}$ \\
\hline Mo et al. 42 & $\begin{array}{l}20 \text { images (DRIVE database }[68 \mid) \\
20 \text { images (STARE database } \mid[36 \mid) \\
28 \text { images (CHASE }|138|)\end{array}$ & No & $\begin{array}{l}A U R O C=0.98, A c c=0.95, S e=0.78, S p=0.98 \\
A U R O C=0.99, A c c=0.97, S e=0.81, S p=0.98 \\
A U R O C=0.98, A c c=0.96, S e=0.77, S p=0.98\end{array}$ \\
\hline Fu et al. 45 & $\begin{array}{l}20 \text { images (DRIVE database }|68| \text { ) } \\
20 \text { images (STARE database } \mid \overline{\mid 136} \text { ) } \\
20 \text { images (CHASE database }|\overline{138}| \text { ) }\end{array}$ & No & $\begin{array}{l}S e=0.76, A c c=0.95 \\
S e=0.74, A c c=0.96 \\
S e=0.71, A c c=0.95\end{array}$ \\
\hline Liskowski et al. 47] & $\begin{array}{l}20 \text { images (DRIVE database }|68|) \\
20 \text { images (STARE database } \mid[36 \mid) \\
28 \text { images (CHASE database } \mid \overline{|138|})\end{array}$ & No & $A U R O C, A c c, S e, S p, k$ \\
\hline Li et al. 48 & $\begin{array}{c}20 \text { images (DRIVE database }|68| \\
20 \text { images (STARE database }|\overline{\mid 136}|) \\
28 \text { images (CHASE }[138 \mid)\end{array}$ & No & $\begin{array}{l}S e=0.76, S p=0.98, A c c=0.96, A U R O C=0.97 \\
S e=0.77, S p=0.98, A c c=0.96, A U R O C=0.99 \\
S e=0.75, S p=0.98, A c c=0.96, A U R O C=0.97\end{array}$ \\
\hline Fraz et al. 62 & $\begin{array}{l}20 \text { images (STARE database [136]) } \\
20 \text { images (DRIVE database }[68 \mid) \\
28 \text { images (CHASE [138]) }\end{array}$ & No & $\begin{array}{l}A U R O C=0.97, A c c=0.95, S e=0.75, S p=0.98 \\
A U R O C=0.98, A c c=0.95, S e=0.74, S p=0.98 \\
A U R O C=0.97, A c c=0.95, S e=0.72, S p=0.97\end{array}$ \\
\hline Orlando et al. $\mid 40$ & $\begin{array}{c}20 \text { images (DRIVE database }[68] \text { ) } \\
20 \text { images (STARE database } \mid 136] \text { ) } \\
20 \text { images (CHASEDB1 database } 138 \mid \text { ) } \\
30 \text { images (HRF database }[139 \mid \text { ) }\end{array}$ & No & $\begin{array}{c}S e=0.79, S p=0.97 \\
M C C=0.75, P P V=0.78 \\
S e=0.77, S p=0.97 \\
M C C=0.74, P P V=0.770 \\
S e=0.73, S p=0.97 \\
M C C=0.70, P P V=0.74 \\
S e=0.79, S p=0.96 \\
M C C=0.69, P P V=0.66\end{array}$ \\
\hline
\end{tabular}

In [65], the feature-based AdaBoost classifier is used to segment retinal vessels. Numerous features are used, such as MF, GF and Gaussian derivatives. However, the most informative ones, according to the authors' analysis, are the second order derivative of Gaussian, multiscale MF using a Gaussian vessel profile, and Staal's ridges [68]. With respect to SVM, AdaBoost relies on the construction of an accurate classification model from a linear combination of weak classifiers, making it easier and faster to train. AdaBoost is also used in [32] and [35] to extract vessel centerline by using convolutional filter and intensity-based feature vector.

Random decision forest (RF) is used in [53] to segment highly tortuous or irregular structures. A modified Gaussianlike bank filter is designed to detect bended tubular structures and the filter outputs are used as features for the classification. When compared to SVM, RF usually has comparable performance with a lower training computational cost. Similarly, RF regressors are used in [34] for the estimation of vessel diameter in coronary artery from CTA. The rationale is evaluating the presence and the degree of stenosis using downstream and upstream properties of coronary tree vasculature as features for the regression.

Inspired by [155], context filters and appearance filters are used in [54]. Optimal Oriented Flux (OOF) [156] is used to exploit vessel appearance information. OOF finds the optimal axis on which image gradients are projected in order to compute the image gradient flux. K-means are used to learn in an unsupervised way a bank of context filters from the OOFfiltered image. RF is used to classify a feature vector made of OOF-output and context filter output. A similar approach is used in [44] using as appearance filter the ridge detector filter defined in [53]. The main advantage of OOF is its robustness against the disturbance induced by closely located adjacent objects. 


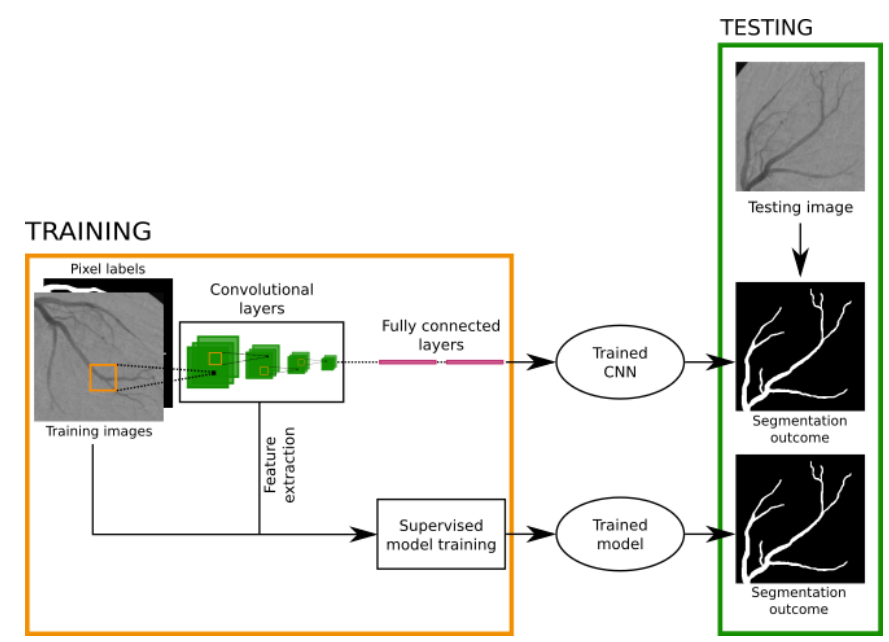

Fig. 4: Convolutional Neural Networks (CNN) for vascular segmentation have been used in two ways: (i) the CNN convolutional layers are used to automatically extract image features, which are then classified with standard supervised learning approaches; (ii) $\mathrm{CNN}$ are directly used to obtain the vascular segmentation by employing fully connected layers.

Neural networks are used in [64] to segment retinal vessels. Gray-level-based and moment invariants-based features are used to train the network, which is defined as a multilayer feedforward network with three hidden layers. The vessel mask is obtained by thresholding the sigmoid output. A further improvement is introduced in [55], where intensitybased and moment-invariant features are used to segment the retinal vasculature through Lattice Neural Network with Dendritic processing (LNND). As a matter of fact, LNND architecture does not require to set the number of hidden layers in the network, allowing for a simple network training and consequently for a reduction of the computational cost.

In the past years, Convolutional Neural Networks (CNNs) have become strongly popular. A CNN is a feed-forward artificial neural network in which the connectivity between its neurons is inspired by the organization of the human visual cortex. The building blocks of a CNN are convolutional and fully connected layers. The convolutional layer parameters consist of a set of filters, whose values are learned during the CNN training. Fully connected layers represent the high-level reasoning block in the CNN. Neurons in a fully connected layer have connections to all activations in the previous layer. CNN-based vascular segmentation workflow is shown in Fig. 4

CNNs have been exploited in [157] to extract esophageal microvessel features from NBI microscopy. The extracted features are then classified with SVM. Similarly, in [56], CNNs are used to extract hierarchical features from retinal color fundus images, which are then classified with ensemble RF. In [58], the feature vector extracted with CNNs is compared with a dictionary feature vector that refers to several vascular patterns. The nearest feature vector extracted from the dictionary, according to the nearest neighbor algorithm, is elected as output vascular pattern. These approaches are particularly useful for small datasets. Indeed, when the dataset variability is small, machine learning approaches, such as SVM and RF, are better suited for achieving pixel classification.

On the other side, CNNs are trained to directly obtain vascular segmentation in [51] for retinal vessel segmentation in OCT angiography, in [38] for carotid segmentation in ultrasound images and in [47] for retinal segmentation in color fundus photography images. Specifically, the CNN fully connected layer is used to classify each pixel in the image as belonging to vessel or background. This approach leads to a fast CNN training, as it already embeds the classification step. However, a large dataset is required for preventing fullyconnected CNN overfitting. Indeed, as already highlighted, if the dataset is small it is recommended to use CNN only to extract features.

In [47] the use of image pre-processing for retinal vessel segmentation with CNN is also investigated. Images are preprocessed with different methods, such as global contrast normalization, zero-phase whitening, data augmentation using geometric transformations and gamma corrections. Authors report an increment in segmentation performance.

Cross-modality learning is used in [48] to segment retinal vessels. The mapping function between the retinal image and the vessel map is learned through a deep neural network.

A unified framework of retinal image analysis that provides both retinal vessel and optic disc segmentation is proposed in [50]. A CNN is designed to segment both retinal vessel and optic disc in single forward pass.

CNNs and Conditional Random Field (CRF) are combined into an integrated deep network called DeepVessel in [45] for retinal vessel segmentation. CRF helps modeling the longrange interactions between pixels and increases the segmentation performance. A similar approach is exploited in [46]. CRF inclusion allows good segmentation performance also in presence of intensity drops and noise.

In [39] CNN is used to produce robust vessel segmentation and tracking in X-ray image sequences. The tracking exploits a multi-dimensional assignment problem, which is solved with rank-1 tensor approximation. Similarly, a deep CNN is trained for estimating local retinal vessel probability via principal component analysis and nearest neighbor search in [52]. The resulting vessel map is exploited to extract the entire connected tree with a probabilistic tracking approach.

A study on the effectiveness of gradient boosting for training CNNs is proposed in [60]. The main benefit of this approach is that both features and the classifier that uses them are learned simultaneously, resulting in a faster procedure that does not require any parameter tuning.

Further developments are presented in [41], [42], where Fully Convolutional Networks (FCN) are used to segment retinal vessels in color fundus photography images. The fully connected layers are replaced by deconvolutional layers allowing to obtain a faster and more precise vessel localization with respect to approaches based on fully connected layer classification. FCN-based vascular segmentation workflow is shown in Fig. 5 .

In [33], the FCN approach is extended to perform 3D vascular segmentation in cardiovascular and pulmonary vessels 


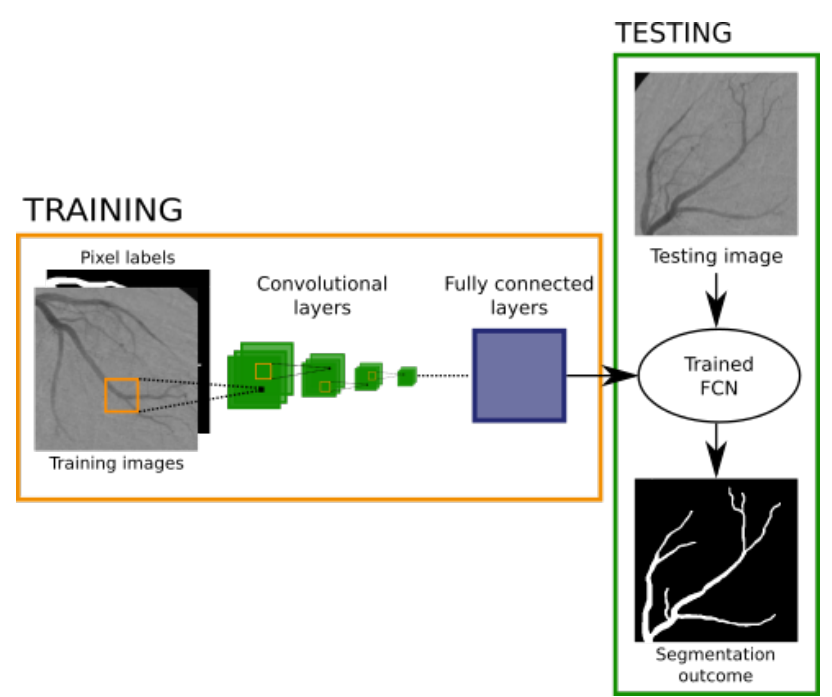

Fig. 5: Fully Convolutional Neural networks (FCN) for vascular segmentation replace fully connected layers with one or more deconvolutional layers, making the segmentation faster.

in MRI and CT volumes, respectively.

In [43], a preliminary attempt of using adversarial learning for vessel segmentation in retinal color fundus photography images is exploited. In adversarial network setup, one network generates candidate segmentations and one evaluates them. With respect to standard network learning, adversarial learning has the potential of improving the segmentation outcome, lowering the number of wrongly classified pixels [158].

A summary of the supervised approaches analyzed above is presented in Table VI

\section{Deformable Model}

Deformable models consider curves or surfaces (S), defined within the image domain, that can move and deform under the influence of internal $\left(\mathbf{F}_{\text {int }}\right)$ and external $\left(\mathbf{F}_{\text {ext }}\right)$ forces. The former are designed to keep $\mathbf{S}$ smooth during the deformation while the latter attract $\mathbf{S}$ toward the vessel boundary. Since $\mathbf{S}$ initialization is required to start the deformation process, a robust deformable model should be insensitive to the initial position, as well as in general to noise. Recent efforts in deformable model formulation focus on easily incorporating in the model formulation both image-guided deformation constraints and a priori clinical knowledge of vessel geometry. This class of algorithms appears suitable to face the segmentation of vessels with complex architecture and high shape and size variability, both in pathological and physiological context. However, the required computational cost in general still represents a limit for real time applications.

Deformable model approaches can be divided in edge-based and region-based, which are hereafter in depth analyzed.

\section{A. Edge-based}

According to the representation of $\mathbf{S}$, edge-based deformable models can be classified in parametric or geometric models [159].

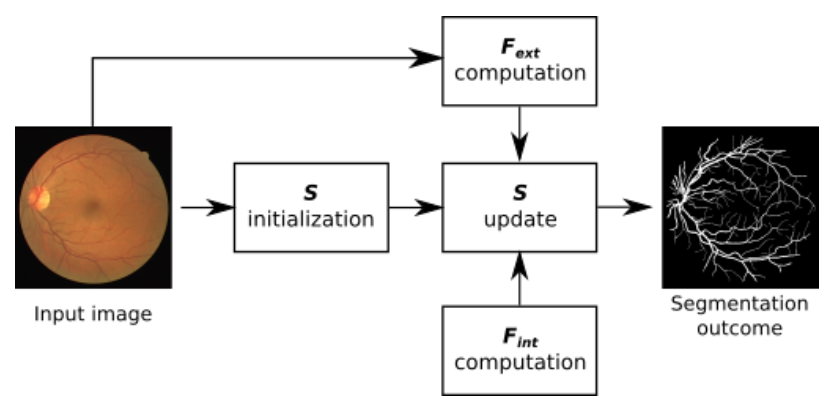

Fig. 6: In parametric deformable model approaches, the segmentation is obtained by evolving a parametrized curve (S) according to external $\left(\mathbf{F}_{\text {ext }}\right)$ and internal forces $\left(\mathbf{F}_{\text {int }}\right)$. $\mathbf{F}_{\text {ext }}$ is formulated according to image-dependent features, such as intensity or gradient. $\mathbf{F}_{\text {int }}$ deals with constraint imposed to the curve evolution, such as curvature and perimeter.

1) Parametric: Parametric deformable models, whose diffusion is mainly due to the work of [160], represent $\mathbf{S}$ in a parametric form. The deformable model problem can be formulated as:

$$
c \frac{\partial \mathbf{S}}{\partial t}=\mathbf{F}_{\text {int }}(\mathbf{S})+\mathbf{F}_{\text {ext }}(\mathbf{S})
$$

being $c$ a damping coefficient. $\mathbf{F}_{\text {int }}$ consists of two main contributions:

$$
\mathbf{F}_{\mathbf{i n t}}=\frac{\partial}{\partial s}\left(p \frac{\partial \mathbf{S}}{\partial s}\right)-\frac{\partial^{2}}{\partial s^{2}}\left(q \frac{\partial^{2} \mathbf{S}}{\partial s^{2}}\right)
$$

with $s \in[0,1]$, and $p$ and $q$ being the weighting parameters that control the $\mathbf{S}$ elasticity and resistance to bending, respectively. The $\mathbf{F}_{\text {ext }}$ term varies according to the method. The general workflow of parametric deformable models is shown in Fig. 6

With respect to geometric models, the main limitation of this class of algorithms, is the difficulty in adapting to changing vessel topology, due to the parametrization of $\mathbf{S}$. Nonetheless, the parametric framework is easy to formulate and allows fast convergence, which is a suitable property to lower computational costs.

In [74], a B-snake active contour [161] is employed to segment retinal vessels. $\mathbf{F}_{\text {ext }}$ consists of (i) a Gradient Vector Flow (GVF) term [162], which describes how the gradient vectors of an image-derived edge-map diffuses inside the image domain, and (ii) a force contributions that impose constrains on the $\mathbf{S}$ evolution, such as vessel cross-section shape, position and size. The good performance, achieved with both low contrasted and thin vessels, deteriorates in presence of pathology, i.e. when the assumptions made on the vessel geometry are not anymore valid.

In [69], an active contour strategy coupled with Kalman filtering is employed to segment the vasculature in CTA. The active contour provides the vessel segmentation in the first CT slice, employing image intensity- and gradient-based $\mathbf{F}_{\text {ext }}$, while the Kalman filtering is used to track the vessel across other CT slices. The Kalman tracking-based approach provides automatic contour initialization, reducing the computational cost with respect to methods based solely on deformable 
TABLE VII: Summary of edge-based parametric deformable model algorithms for blood vessel segmentation (for performance indexes refer to Tab. IIII). CTA: Computer Tomography Angiography.

\begin{tabular}{|c|c|c|c|}
\hline Method & Testing dataset & Synthetic data & Segmentation performance measure \\
\hline Lee et al. 69 & CTA & Yes & $S e=0.95, S p=0.99$ \\
\hline Cheng et al. 74 & $\begin{array}{l}3 \text { Abdominal, 1 Carotid } \\
\text { 2 Lung } \\
\& 24 \text { CTA volumes (ROTTERDAM database }[144])\end{array}$ & Yes & $\begin{array}{c}D S C=0.86 \\
\text { Visual } \\
O V=0.93, O F=0.53, O T=0.94\end{array}$ \\
\hline Zhang et al. |76| & 20 images (DRIVE database $[68 \mid$ ) & No & $A c c=0.95, S e=0.75, S p=0.96, A U R O C=0.95$ \\
\hline
\end{tabular}

TABLE VIII: Summary of edge-based geometric deformable model algorithms for blood vessel segmentation (for performance indexes refer to Tab. III]. CT: Computed Tomography, CTA: Computer Tomography Angiography, MRA: Magnetic Resonance Angiography, US: Ultrasound.

\begin{tabular}{cccc}
\hline Method & Testing dataset & Synthetic data & Segmentation performance measure \\
\hline Law et al. [71] & 1 CTA, 3 MRA & Yes & Visual \\
Moreno et al. [72] & 18 CTA (CASDQEF database [143]) & Yes & Visual \\
Wang et al. [73] & 24 CTA volumes (CASDQEF database [143]) & No & DSC $=0.69$ \\
Zhu et al. [75] & 10 CT volumes & No & US \\
Patwardhan et al. [7] & 7 MRA volumes & Yes & Visual \\
Valencia et al. [70] & & Yes & Visual \\
\hline
\end{tabular}

models. Similarly, in [77], a single spatial Kalman-filter tracker keeps track of the vessel center-line in 3D ultrasound. The vessel boundaries are then estimated by growing an area weighted active contour outward from the centerline.

In [76], active contours are used to segment retinal vessels. A rough vessel edge map is computed by thresholding the vesselness measure defined in [124]. The vessel edges are then used to initialize two active contours $\mathbf{S}_{-}$and $\mathbf{S}_{+}$. The active contours deform according to intensity-based $\mathbf{F}_{\text {ext }}$. A further constraint is introduced in the $\mathbf{F}_{\text {ext }}$ formulation, to avoid the intersection of $\mathbf{S}_{-}$and $\mathbf{S}_{+}$, by controlling their relative distance. The vesselness measure allows for the automated initialization of the model, while the intensity-based formulation of the $\mathbf{F}_{\text {ext }}$ addresses the problem of detecting small and peripheral vessels.

A summary of the analyzed parametric deformable approaches is presented in Table VII.

2) Geometric: Geometric deformable models, first introduced in [163] and in [164], are based on the curve-evolution theory, described by:

$$
\frac{\partial \mathbf{S}}{\partial t}=Z \mathbf{n}
$$

being $Z$ a function called speed function and $\mathbf{n}$ the unit normal to $\mathbf{S}$.

Two main terms can contribute to the speed function formulation [165]: (i) a constant term, which defines the deformation along the $\mathbf{S}$ normal through a coefficient $\nu$, and (ii) a deformation term that depends on the $\mathbf{S}$ curvature $(\zeta)$. The speed

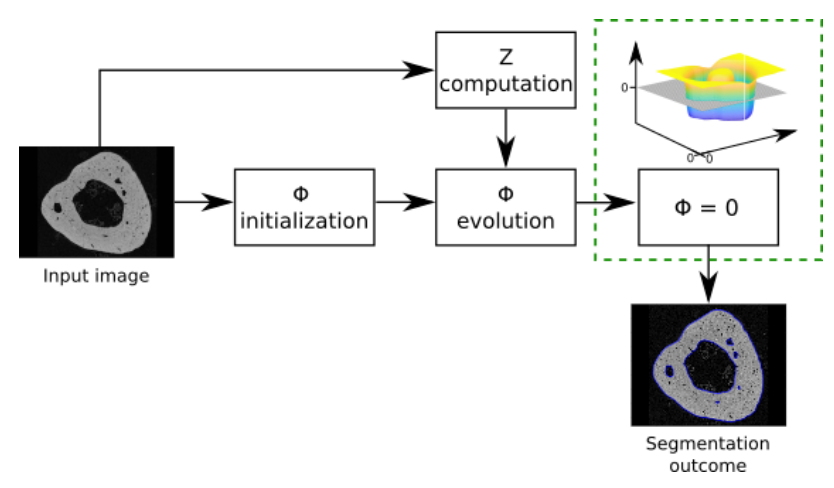

Fig. 7: In geometric deformable model approaches, segmentation is treated as a curve evolution problem. The evolution is implemented using the level set method, which needs to define a level set function $(\Phi)$. After $\Phi$ initialization, $\Phi$ evolution is controlled by the speed function $(Z)$, which can be defined for example according to intensity or gradient-based information. Once the $\Phi$ evolution ends, the segmentation contour corresponds to the zero-level of $\Phi$.

function formulation defines difference among methods. Since no curve-parametrization is introduced, the evolution of $\mathbf{S}$ only depends on geometrical constraints. Consequently, topology changes can be easily handled, overcoming the main limits of parametric models.

The geometric curve evolution is commonly implemented 
TABLE IX: Summary of region-based deformable model algorithms for blood vessel segmentation (for performance indexes refer to Tab. III). CTA: Computed Tomography Angiography, IR: Infrared, MRA: Magnetic Resonance Angiography, DSA: Digital Subtraction Angiography, US: Ultrasound.

\begin{tabular}{|c|c|c|c|}
\hline Method & Testing dataset & Synthetic data & Segmentation performance measure \\
\hline Tian et al. 79 & $\begin{array}{l}2 \text { DSA, } 1 \text { IR, } \\
2 \text { MRA, } 1 \text { US }\end{array}$ & Yes & Root mean squared error \\
\hline Klepaczko et al. 78 & MRA volumes & Yes & Visual \\
\hline Law et al. 80 & 4 MRA volumes & Yes & Visual \\
\hline Liang et al. 82 & 54 Microscopy images & No & $\begin{array}{c}P P V=0.96, S e=0.87 \\
D S C=0.91\end{array}$ \\
\hline Wang et al. $\mid 81$ & - & Yes & Visual \\
\hline Law et al. 87 & 1 image (DRIVE database $\overline{68}$ ) & No & $\begin{array}{l}\text { Visual } \\
\text { Visual }\end{array}$ \\
\hline Wang et al. 85 & 20 images (STARE database [136]) & No & $\begin{array}{c}A c c=0.94, S e=0.76, S p=0.96, \\
A U R O C=0.86\end{array}$ \\
\hline Xiao et al. 86 & $\begin{array}{l}20 \text { images (STARE database }|136|) \\
20 \text { images (DRIVE database }\end{array}$ & No & $\begin{array}{l}A c c=0.95, S e=0.71, S p=0.97 \\
A c c=0.95, S e=0.75, S p=0.98\end{array}$ \\
\hline Zhao et al. 84 & $\begin{array}{l}20 \text { images (STARE database }[136 \text { ) } \\
20 \text { images (DRIVE database } 68 \text { ) }\end{array}$ & No & $\begin{array}{c}S e=0.74, S p=0.98, A c c=0.95 \\
A U R O C=0.86 \\
S e=0.78, S p=0.98, A c c=0.96 \\
A U R O C=0.87\end{array}$ \\
\hline Zhao et al. 83 & $\begin{array}{c}20 \text { images (STARE database } 136] \text { ) } \\
20 \text { images (DRIVE database }|68| \text { ) } \\
143 \text { images (ARIA 137|) } \\
8 \text { images (VAMPIRE http://vampire.computing.dundee.ac.uk) }\end{array}$ & No & $\begin{array}{c}S e=0.79, S p=0.97, A c c=0.95 \\
A U R O C=0.88 \\
S e=0.74, S p=0.98, A c c=0.95, \\
A U R O C=0.86 \\
S e=0.75, S p=0.93, A c c=0.94, \\
A U R O C=0.84 \\
S e=0.72, S p=0.98, A c c=0.98, \\
A U R O C=0.85\end{array}$ \\
\hline
\end{tabular}

using the Level Set (LS) method [166]:

$$
\frac{\partial \Phi}{\partial t}=Z(\zeta)|\nabla(\Phi)|
$$

being $\Phi=\Phi(\mathbf{S})$ the LS function.

The geometric deformable model workflow is shown in Fig. 7.

A rather popular LS formulation, proposed in [167] and in [168], introduces a regularization term to deal with leaky vessel edges and possible gaps:

$$
\frac{\partial \Phi}{\partial t}=Z(\zeta)|\nabla(\Phi)|+\nabla Z \nabla \Phi
$$

Different speed functions are used in the literature, mainly based on gradient information as in [169], [170], [171], [172].

In [73] LS is used to segment coronary artery lumens in CTA. The speed function (Eq. 17p) is made of three contributions: the $\Phi$ minimum curvature $\left(z_{\zeta}\right)$, an image intensity-based term $\left(z_{\text {image }}\right)$, and a model-based term $\left(z_{\text {model }}\right)$ that measures the deviation of the evolving curve from a cylindrical model, whose axis corresponds to the vessel centerline.

$$
Z=z_{\zeta}+z_{\text {image }}+z_{\text {model }}
$$

During the $\Phi$ evolution, the vessel centerline is iteratively computed with the Fast Marching Method (FMM) [173].

The LS function $\Phi$ for vessel lumen segmentation described in [73] acts as initialization for the evolution of a second LS function $\Phi_{\text {outer }}$, which provides the vessel outer layer segmentation [72]. A modified version of Eq. 17 is used to define the speed function of $\Phi_{\text {outer }}$. The $z_{\zeta}$ is replaced by the Laplacian of the mean curvature, as suggested in [174], and a $z_{\text {lumen }}$ term is introduced to avoid the vessel outer layer to shrink inside the vessel lumen, by pushing $\Phi_{\text {outer }}$ outwards from the vessel lumen.

In [75], a LS algorithm is implemented to segment pulmonary vasculature. The employed speed function (Eq. 18, inspired by [167], is modified to include the vesselness measure $(V)$ defined in [124]. $V$ is built from the eigenvalues of the image Hessian and represents the likelihood of a pixel to be a part of a vessel.

$$
\begin{array}{r}
Z=\frac{1}{1+|\nabla G(\sigma) * V|}(\nu-\epsilon \zeta) \\
\epsilon=m e^{-n V^{3}}
\end{array}
$$

being $G$ a Gaussian kernel with standard deviation $\sigma, \nabla$ the 
gradient operator, and $m$ and $n$ two constant coefficients.

In this way, the typical high vessel $\zeta$ does not penalize the LS evolution, resulting in a faster segmentation. Moreover, the use of the vesselness enhanced vasculature allows for a better treatment of image inhomogeneities.

In [71], gradient flux symmetry along the vessel centerline and gradient flux asymmetry along vessel edges are imposed to drive an active contour model. Gradient flux-based algorithms are quite popular (e.g. [175], [176], [177], [178], [179], [180]) since gradient flux allows overcoming intensity inhomogeneities, resulting in the segmentation of the entire vascular tree without contour leakages.

In [70] a generalized cylinder deformable model is encoded in the LS formulation to extract the vasculature from MRA and simulate its interaction with a stent. The model internal deformation forces takes into account the particular global cylindrical shape and the expected geometric properties of a vessel to improve the segmentation accuracy.

A summary of the analyzed geometric deformable model approaches is presented in Table VIII.

\section{B. Region-based}

Region-based deformable models were first introduced in [181] by considering the deformable curve $\mathbf{S}$ as moving under image foreground and background region constraints. Both regions are considered as statistically homogeneous, and the main differences among this class of algorithms rely in the definition of the region statistics. Region-based deformable models were introduced to overcome some of the edge-based deformable model issues, as the boundary leakage problem, that arises especially when dealing with noisy images or non-uniform intensity. Moreover, since edge-based deformable models are mainly driven by intensity or gradient derived external forces, the segmentation becomes challenging if the curve initialization is performed far from the boundary of interest, due to the local nature of the intensity gradient [85]. Despite the fact that region-based deformable models benefit of adaptive topology, it is worth noting that this comes at the expense of higher computational cost with respect to edgebased deformable models [182].

Region-based deformable models define an energy minimization problem, which is solved employing LS. The energy formulation for an image $I=I(\mathbf{r})$ is:

$$
\begin{array}{r}
E=c_{l} \text { length }(\mathbf{S})+c_{a} \text { area }(\mathbf{S})+e_{1} E_{1}(\mathbf{S})+e_{2} E_{2}(\mathbf{S}) \\
\text { with } \\
E_{1}(\mathbf{S})=\int_{\text {inside }(\mathbf{S})}\left|I(\mathbf{r})-c_{1}\right|^{2} d \mathbf{r} \\
E_{2}(\mathbf{S})=\int_{\text {outside }(\mathbf{S})}\left|I(\mathbf{r})-c_{2}\right|^{2} d \mathbf{r}
\end{array}
$$

being $c_{l}, c_{a}, e_{1}$ and $e_{2}$ constant parameters and $c_{1}$ and $c_{2}$ the image intensity average inside and outside $\mathbf{S}$, respectively. This energy formulation is globally defined and local information is not considered. To overcome this limit, a localized region energy formulation is introduced in [183], by replacing $E_{1}$ and $E_{2}$ with $E_{1}^{l o c}$ and $E_{2}^{l o c}$ :

$$
\begin{aligned}
E_{1}^{l o c}(\mathbf{S}) & =\int\left[\int_{\text {inside }(\mathbf{S})} w(\mathbf{r}-\mathbf{y})\left|I(\mathbf{y})-f_{1} I(\mathbf{r})\right|^{2} \mathbf{d y}\right] \mathbf{d} \mathbf{r} \\
E_{2}^{l o c}(\mathbf{S}) & =\int\left[\int_{\text {outside }(\mathbf{S})} w(\mathbf{r}-\mathbf{y})\left|I(\mathbf{y})-f_{2} I(\mathbf{r})\right|^{2} \mathbf{d y}\right] \mathbf{d r}
\end{aligned}
$$

being $w$ a weighting function, and $f_{1}$ and $f_{2}$ functions that approximate the image intensity inside and outside $\mathbf{S}$, respectively. Following a similar approach, in [79] the localized region energy formulation is used $(w=G(\sigma))$, and a further energy contribution term is built from the vesselness measure in [124].

The active contour formulation in [181] is combined with phase-based vessel enhancement and intensity inhomogeneity correction (used as region information) in [83] to segment both color fundus photography and fluorescein angiography images. Similarly, simulated MRA images are segmented in [78]. The enhancement and correction strategies are particularly useful for small branching vascular structure detection.

In [81], the local statistic of pixel intensities is estimated with a Gaussian probability distribution $p$, with $E_{1}^{l o c}$ and $E_{2}^{l o c}$ defined as in Eq. 21 .

$$
\begin{array}{r}
E_{1}^{l o c}(\mathbf{S})=-\int\left[\int_{\text {inside }(\mathbf{S})} w(\mathbf{r}-\mathbf{y}) \log p_{1}(I(\mathbf{y})) \mathbf{d} \mathbf{y}\right] \mathbf{d} \mathbf{r} \\
E_{2}^{l o c}(\mathbf{S})=-\int\left[\int_{\text {outside }(\mathbf{S})} w(\mathbf{r}-\mathbf{y}) \log p_{2}(I(\mathbf{y})) \mathbf{d} \mathbf{y}\right] \mathbf{d} \mathbf{r} \\
\text { with } \\
p_{k}(I(\mathbf{y}))=\frac{1}{\sqrt{2 \pi} \sigma_{k}(\mathbf{x})} e^{-\frac{\left(I(\mathbf{y})-\mu_{k}(\mathbf{r})\right)^{2}}{2 \sigma_{k}(\mathbf{r})^{2}}}
\end{array}
$$

being $\mu_{k}$ and $\sigma_{k}(k=1$ inside $(\mathbf{S})$ and $k=2$ outside $(\mathbf{S}))$ the local mean and standard deviation of the pixel intensity.

A further improvement is introduced in [85] by considering also the local statistic of the image vesselness map defined in [184].

In [86], spatial constraints are introduced in the estimation of the local pixel intensity distribution. Each pixel classification depends on its neighborhood, with the main scope of privileging connected set of pixels in order to increase the segmentation robustness.

Local phase map and pixel intensity are used as region information in [84]. The former allows obtaining an accurate vessel map, the latter lowers false positive vessels.

In [87], [80], the vessel boundary orientation is locally estimated based on the orientation that minimizes the weighted local variance and is used to drive an active contour model. Region-based deformable model algorithms are summarized in Table IX

\section{TRACKING}

Blood vessel tracking algorithms usually consist in the definition of seed points followed by a growth process guided by image-derived constraints. Seed points can be either manually defined or obtained through vessel enhancement approaches. 
TABLE X: Summary of tracking algorithms (for performance indexes refer to Tab. III). CTA: Computer Tomography Angiography, 3DRA: 3D Rotational Angiography, CFP: Color Fundus Photography, MIP: Maximum Intensity Projection, MRA: Magnetic Resonance Angiography, US: Ultrasound.

\begin{tabular}{|c|c|c|c|}
\hline Method & Testing dataset & Synthetic data & Segmentation performance measure \\
\hline Turetken et al. 115 & 2 Microscopy & No & Visual \\
\hline Yureidini et al. $[90$ & 10 3DRA volumes & No & Average symmetric surface distance $|185|<1$ \\
\hline Shin et al. 95 & 18 Fluoroscopic x-ray images & No & $S e=0.84, A c c=0.9$ \\
\hline Amir-Khalili et al. 97] & $8 \mathrm{US}$ & Yes & $A U R O C=0.95$ \\
\hline Tang et al. [101] & 38 MRI volumes & No & $D S C=0.89$ \\
\hline Wink et al. 105 & 32 MRA & No & Average distance $=0.65 \mathrm{~mm}$ \\
\hline Robben et al. 88 & 50 MRA volumes & No & $A c c=0.94, P P V=0.96, S e=0.96$ \\
\hline Rempfler et al. 89 & 5 micro MRA volumes & No & $A c c=0.52$ \\
\hline Cetin et al. 91$]$ & $\begin{array}{l}50 \text { MRA volumes } \\
24 \text { CTA }\end{array}$ & $\begin{array}{l}\text { No } \\
\text { No }\end{array}$ & $\begin{array}{l}D S C=0.93 \\
D S C=0.97\end{array}$ \\
\hline Carrillo et al. 96 & $\begin{array}{l}16 \text { MRA volumes } \\
12 \text { CTA }\end{array}$ & $\begin{array}{l}\text { No } \\
\text { No }\end{array}$ & $\begin{array}{c}F N=0.20, F P=0 \\
F N=0.28, F P=0.08\end{array}$ \\
\hline Li et al. $\mid 104$ & $2 \mathrm{MRA}$ volumes and $2 \mathrm{CTA}$ & Yes & $D S C$ \\
\hline Shim et al. 93 & 15 CTA volumes & No & $S e=0.87$ \\
\hline Cherry et al. 94 & $30 \mathrm{CTA}$ volumes & No & $S e=0.68, A c c=0.75$ \\
\hline Benmansour et al. 98 & $56 \mathrm{CTA}$ volumes & Yes & $\begin{array}{c}D S C=0.84, D_{\text {msd }}[186=0.80, \\
\left.D_{\text {rmssd }} \mid 186\right]=1.57, D_{\max }=6.47[186\end{array}$ \\
\hline Biesdorf et al. 99 & $10 \mathrm{CTA}$ volumes & Yes & $D S C=0.97$ \\
\hline Lugauer et al. [100] & 30 CTA volumes (ROTTERDAM database [144|) & No & $D S C=0.76$ \\
\hline Wang et al. 102 & 32 CTA volumes (ROTTERDAM database [144|) & No & $O V=0.81$ \\
\hline Friman et al. 103 & $\begin{array}{c}32 \text { CTA volumes (ROTTERDAM database } 144 \text { ) } \\
\text { \& liver volumes }\end{array}$ & Yes & $O V=0.99$ \\
\hline Cetin et al. 92 & 32 CTA volumes (ROTTERDAM database [144|) & Yes & $O V=0.96$ \\
\hline Zeng et al. $\mid 106$ & 6 CTA & Yes & $A c c=0.98, S e=0.8, S p=0.99$ \\
\hline Bauer et al. 107] & 15 contrast enhanced CT & Yes & $\mathrm{FN}=0.26 \%$ \\
\hline Amir-Khalili et al. [108] & 15 Endoscopy images & No & $A U R O C=0.72$ \\
\hline Chen et al. 110 & 2 CFP images and $2 \mathrm{MRA}$ & Yes & Visual \\
\hline Bhuiyan et al. $\mid 111]$ & 44 CFP images & No & $A c c=0.88$ \\
\hline Liao et al. 112] & 4 CFP images (DRIVE database $[68 \mid$ ) & No & $A c c=0.99$ \\
\hline Rouchdy et al. [113] & 20 CFP images (DRIVE database [68) & Yes & $D S C=0.77, S e=0.66, S p=0.91$ \\
\hline Stuhmer et al. $\mid 114$ & 20 CFP images (DRIVE database 68 ) & No & $A c c=0.94, S e=0.84, S p=0.96$ \\
\hline Delibasis et al. 118 & 20 CFP images (DRIVE database 68 ) & No & $\mathrm{Se}, \mathrm{Sp}, \mathrm{Acc}, \mathrm{ROC}$ curve \\
\hline Liao et al. $\mid 116$ & 10 CFP images (STARE database $[136 \mid$ ) & No & Visual \\
\hline Kaul et al. [117] & 3 retinal images & Yes & $T P=0.90, F P=0.15, F N=0.10$ \\
\hline
\end{tabular}


TABLE X Summary of tracking algorithms (for performance indexes refer to Tab. III). CTA: Computer Tomography Angiography, 3DRA: 3D Rotational Angiography, CFP: Color Fundus Photography,MIP: Maximum Intensity Projection, MRA: Magnetic Resonance Angiography, US: Ultrasound.

\begin{tabular}{|c|c|c|c|}
\hline Method & Testing dataset & Synthetic data & Segmentation performance measure \\
\hline Breitenreicher et al. $\mid 119$ & - & No & - \\
\hline Benmansour et al. 120 & - & Yes & Visual \\
\hline Wink et al. $\mid 121$ & - & Yes & Visual \\
\hline Chen et al. [109] & - & No & Visual \\
\hline
\end{tabular}

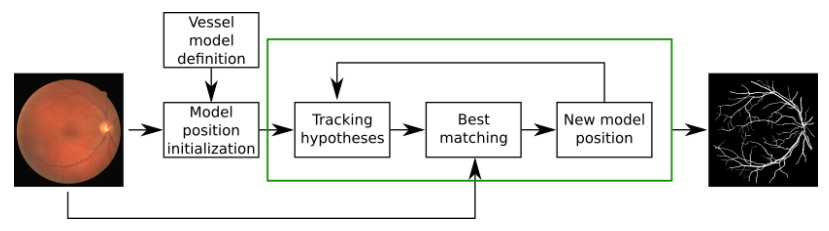

Fig. 8: Model-based tracking approaches require to define a vessel model, which most of the time takes the form of a cylinder. After model initialization, at each tracking step the new model position is obtained by finding the best imagemodel match among all the possible new model positions (tracking hypotheses). The match is computed based on image features computed in a neighborhood of the current model position.

Tracking approaches are particularly useful to segment connected vascular trees, for which the segmentation can be achieved using a limited number of seed points. Differences between tracking-based methods lie in the definition of the tracking constraints. A classification can be done considering model-based tracking approaches, which track vessels according to a vascular model, and minimum cost path approaches, which finds the minimum path between two seed points according to image-derived metrics.

\section{A. Model-based}

Model-based tracking approaches, usually exploited for 3D vessel segmentation, address the segmentation problem as the tracking of a predefined model in 3D volumes. The model can assume different shapes, even if the most commonly adopted is a 3D cylinder with elliptical or circular section, the latter of which is usually preferred when dealing with small vessels. The starting position and orientation of the model are defined by placing an initial seed and estimating the vessel direction in the seed, which can be done either manually or automatically, e.g. exploiting filtering approaches such as MF. At each tracking step, the next model position and orientation are computed locally as the best match between the model itself and the image data in the current model neighborhood. Both deterministic and statistical approaches can be used to investigate the possible vessel trajectories, and different imagederived features, e.g. intensity or gradient flux, can be used to compute the match between the image and the template. The workflow of model-based segmentation algorithms is shown in Fig. 8 The main limit of this class of algorithms is the early termination in presence of intensity inhomogeneities, noisy images, and when the model is not able to replicate the vessel architecture, e.g. in presence of pathology.

Recursive tracking is performed in [96] by recruiting pixels according to a cluster algorithm based on geometry and intensity constraints. Cylindrical geometry is assumed for the tracking. The clustering minimizes the intra-class intensity variances and the inertia moment of the vessel class, enhancing the cylindrical structures.

A deterministic tracking approach is proposed in [103 to segment coronaries in CT, exploiting Multiple Hypothesis Template Tracking (MHTT). At each tracking step, a range of possible cylindrical model predictions is geometrically computed, exploring a user-defined number of directions equidistributed on the half-unit sphere centered at the current model position. Instead of retaining only the absolute best match, which not always represents the correct tracking (e.g. in presence of bifurcation, intensity inhomogeneities and noise), MHTT allows considering multiple possible vessel trajectories by building a trajectory search tree. The leaf of the tree that globally guarantees the higher matches provides the segmentation. The method shows high accuracy in extracting and preserving small and weakly contrasted vessel centerline, and improving the bifurcation detection, due to both the adopted vessel model and to the MHTT searching approach.

In [102], coronary arteries are tracked with a statistical bayesian approach in CTA. The model matching is evaluated according to cylindrical minimal flux [187], which measures the inward gradient flux through a cylindrical surface. The inward flux is maximized when the cylinder is aligned with the coronary.

Other common statistic approaches, which focus on solving non-linear Bayesian state estimation problem for vessel tracking, use Particle Filtering (PF) [188]. PF is employed for tracking cerebral vessels in [93] and for marginal artery localization in colonography [94].

Vasculature tracking in 3D Rotational Angiography (3DRA) is performed in [90]. The main innovation consists in the inclusion of the RANdom SAmple Consensus (RANSAC) [189]. RANSAC is an iterative method used to estimate the quality 
of the vascular model fitting from the set of observed data. RANSAC allows handling possible outlier pixel values, making the tracking more robust to noise and intensity drop. The algorithm succeeds in tracking the centerline of vessels with complex architecture, overcoming the problem of bifurcation and vessel kissing.

Cylindrical-model tracking with MRF is exploited in [100]. A discrete number of radial candidates along equiangular rays in slices orthogonal with respect to an initialized vessel centerline are considered as random variables of a MRF graph. The algorithm performance is boosted by including supervised PBT to estimate vessel boundaries. A similar approach is exploited in [95], where otimal correspondences between consecutive frames of fluoroscopic X-ray sequences are determined within a MRF optimization framework.

In [107], vessel skeletons are extracted by using cylindrical structures enhancing filter with constraints on vessel radius and centerline direction. Vessel segmentation is achieved using the graph cut algorithm [190]. With graph cut, the segmentation problem is interpreted as image partitioning in two different categories (i.e. vessel and background). The cut of the graph, obtained by minimizing a cost term that depends on image gradient and vessel shape priors, gives the partition of the graph vertices into the vessel and background subsets. A similar approach is exploited in [111], where false vessels are clarified imposing a threshold on the maximum possible distance between vessel edges.

An intensity-based vessel model is defined in [92] to extract coronaries in CTA volumes. A second order tensor is constructed from image intensity and used to drive the segmentation evolution. A similar approach with higher-order tensors is used in [91]. The use of the second order tensor allows dealing with complex structures, such as bifurcations.

A parametric vascular model that can assume arbitrarily complex shape is used in [118] to segment retinal vessel. From the vessel model, vessel diameter can be retrieved in a supervised way at each detected vessel centerline pixel after tuning the vessel model-to-diameter transformation on a training set. Thus, this approach can easily adapt to different vessel topologies as long as a proper training dataset is used. Similarly, in [99], the problem of estimating the shape of a vessel is treated as a convex parameter estimation problem. Vessel tracking is performed according to the locally estimated vessel direction and radius.

In [97], [108] the monogenic signal [191] is used to estimate the image motion vector field and track the temporal displacement of vessels in endoscopic images. The vessels are detected where the divergence of the motion vector field is high. By including motion feature, the method exploits the vascular pulsatile behavior, allowing a targeted discrimination between vessels and background.

\section{B. Minimum cost path}

A Minimum Cost Path (MCP) is a curve $\left(C_{\mathbf{p}_{1}, \mathbf{p}_{2}}\right)$ connecting two usually manually defined points, $\mathbf{p}_{1}$ and $\mathbf{p}_{2}$, that minimizes the energy functional:

$$
E(\gamma)=\int_{\gamma} P\left(\gamma(s), \gamma^{\prime}(s)\right) d s
$$

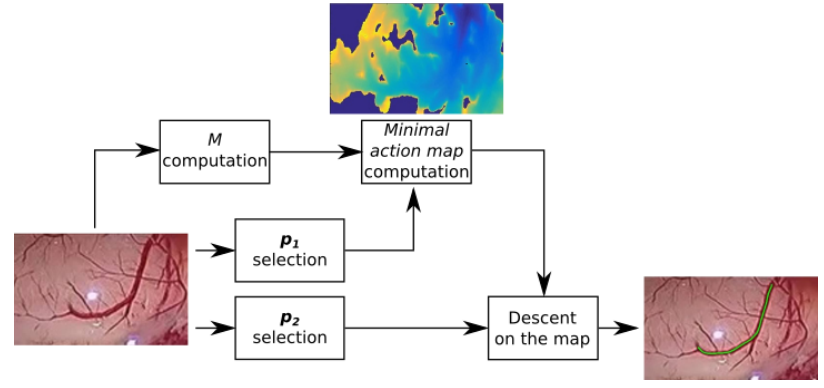

Fig. 9: The minimum cost path algorithm finds the shortest path between two points $\left(\mathbf{p}_{\mathbf{1}}\right.$ and $\left.\mathbf{p}_{\mathbf{2}}\right)$ by minimizing an energy functional that depends on a metric tensor $(M) . M$ is defined according to image features, such us intensity, gradient or higher-order derivatives. Moreover, $\mathbf{p}_{\mathbf{1}}$ is the only global minimum of $M$. Energy minimization is solved by computing the minimal action map (e.g. by using fast marching method). Once the map is computed, the path between $\mathbf{p}_{\mathbf{1}}$ and $\mathbf{p}_{\mathbf{2}}$ can be retrieved with the gradient descent on the map starting from $\mathbf{p}_{2}$.

where $P\left(\gamma(s), \gamma^{\prime}(s)\right)=\sqrt{\left.\gamma^{\prime T}(s) M(\gamma(s)) \gamma^{\prime}(s)\right)}$ is an infinitesimal distance path computed according to the metric tensor $M$ (symmetric and positive-definite). The MCP formulation can be either isotropic or anisotropic, according to the formulation of $M$. The energy minimization problem is solved through the computation of the minimal action map, whose values can be interpreted as the arrival time of a propagation front that starts from $\mathbf{p}_{\mathbf{1}}$, which is the only action map global minimum, and moves with velocity dependent on $M$, as demonstrated in [192]. The work proposed in [192] is employable on 2D structures, while further extensions introduced in [193], [194] lead to the segmentation of 3D structure. The minimal action map can be computed using different FMM formulations, according to the nature of $M$. The FMM formulation for isotropic $M$ was introduced in [195] and [196], and improved in many subsequent works to provide enhanced computational efficiency and accuracy, e.g. in [197]. On the other hand, FMM schemes such as those presented in [198], [199], [200] can be used to deal with anisotropic $M$. $C_{\mathbf{p}_{1}, \mathbf{p}_{2}}$ can be found using gradient descent method on the minimal action map from $\mathbf{p}_{\mathbf{2}}$ to $\mathbf{p}_{\mathbf{1}}$. Gradient descent method can be solved with standard numerical methods, such as [201]. The workflow of a typical MCP segmentation algorithm is shown in Fig. 9 .

In [101], carotids are segmented in multispectral MRI. The MCP between two manually defined seed points (a start and an end point) is found accordingly to a metric based on image gradient [202] and intensity [203]. The centerline is then used to initialize a geodesic deformable model [167] with intensitybased speed function. Multi-scale Hessian-based enhancement filtering is used to drive the path evolution in [121], [105], allowing higher robustness to noise with respect to using simple gradient information.

In [98], OOF [156] is used to obtain an estimation of the carotids direction and radius, which is then used to construct $M$. In [106], OOF [156] and oriented flux antisym- 
metry are combined to detect liver vessels. Once the vessel centerlines is extracted, an intensity model based on FMM and integrated into graph cuts is applied to obtain the segmentation. The automatic centerline extraction results to be effective in presence of small, thin and overlapping vessels.

A front propagation method for vessel segmentation with the dynamic anisotropic Riemannian metric and anisotropic FMM is proposed in [109]. The Riemannian metric is defined using a prior estimate of vessel orientation, detected by the OOF filter, and the local intensity values.

Geodesic voting is introduced in [113]. With respect to classical MCP algorithm, here multiple end points are defined. The potential that drives the geodesic evolution from the manually defined start point and the end points assumes low value in correspondence to the target vascular tree, leading to a high geodesic density and high vote. LS is used to propagate the centerline and obtain the segmentation.

MCP is used in [119] to segment vessel centerline after having enhanced vessels with a multi-scale tubular structure enhancement filter. A learning-based branch classifier is used as post-processing to remove false positive vessels. A similar approach is exploited in [114], where the shortest path energy minimization is driven by image gradient and intensity. Connectivity priors on vessel tree geometry are included to lower false positive vessels.

Geodesic are integrated in a graph-based framework in [88], [115]. The main innovation is allowing cycles inside the graph, avoiding early-termination due to bifurcations or vessel kissing. A similar approach is exploited in [89], where the computation of the most probable path in the graph also takes into account geometric distributions computed from training samples. The main advantage of this method is the inclusion of geometrical-physiological prior knowledge, which allows improving the overall segmentation quality.

In [112], curvature regularization of the local path is dynamically included in the FMM formulation. The main innovation is the extension of the FMM formulation with dynamic speed computed at each path evolution step, inspired by [116].

MCP with keypoint detection is used in [109], [120], [117], [104], [110]. Instead of retrieving the MCP between the source and the end point, new intermediate points, called keypoints, are found imposing constraints on the length of the path between consecutive keypoints. This significantly improves the MCP robustness to noise. MCP is then applied to find the shortest path between consecutive keypoints.

A summary of the analyzed tracking approaches is presented in Table $\mathrm{X}$

\section{CONCLUSION}

This review presented a detailed analysis of a wide spectrum of the most modern vessel segmentation techniques. These included image processing methods based on machine learning, deformable models and tracking approaches.

Vessel segmentation dates back to mid 1900s and a solid and rich literature now exists in this field. However, despite the efforts and the already achieved results, there are still opportunities for improvements.
- The absence of a systematic evaluation workflow emerges as a first critical point in the vessel segmentation literature. It can be difficult to compare algorithm performances if the reported metrics are not consistent. Moreover, in most cases algorithms are tested on dataset not publicly available for the community, making the inter-algorithm comparison almost impossible.

- Regarding the GS definition for segmentation quality assessment, it would be of interest reporting the number of clinicians who perform the GS segmentation, as well as their degree of expertise. In case of multiple clinicians, also the way of combining the single GS should be reported. In this way, algorithm performance could be analyzed more robustly, e.g. considering the dispersion of the GS segmentations, symptomatic of the degree of image complexity.

- As presented, a large range of methods has been developed and enhanced over the years for segmenting blood vessels on medical images. However, none of them are appropriate for all applications. Processing frameworks are still developed on an ad-hoc fashion since each application presents its own specific requirements. These are given by characteristics such as the anatomical region of interest, image acquisition method, noise levels, illumination, etc. In fact, image quality strongly affects segmentation performances and a well performing method in one context may not be that appropriate in another contexts. This represents a further limitation in our ability to compare across different methods.

- One of the main issues still remaining is the segmentation of pathological vessels. Unfortunately not much research effort has been dedicated to this issue yet. Research is needed since some of the main assumptions made for healthy vessels (such as linearity and circular crosssection) do not hold in pathological tissues, requiring new vessel model formulations.

- The constant development of diagnostic imaging systems is also providing even more detailed and higher resolution images of blood vessels, resulting in processing algorithms with higher and higher computational cost. At the same time, many clinical applications require real time processing. This issue can be faced with parallel implementation and the use of Graphic Processor Unit (GPU), however few of the analyzed researches have directly focused on this. A review on medical image segmentation on GPUs can be found in [204].

- Deep learning algorithms for vessel segmentation are becoming strongly popular. With respect to machinelearning algorithms, where the feature extraction process requires strong domain expertise to understand which are the most suitable features, deep learning directly extracts a suitable internal representation of the image. Deep learning is taking advantage of the increasing computational power (e.g. GPU) as well as of data availability.

- Deep learning via unsupervised or semi-supervised learning is becoming a topic of interest to overcome the lack of properly annotated vessel images [205], [206]. Deep learning with reinforcement [207], generative net- 
works [158] and recurrent networks [208] are also becoming popular, despite direct application on vessel segmentation have not been proposed yet.

- Vessel data availability is increasing thanks to the spread of diagnostic imaging tools. Such amount of data, if shared, represents a possibility of building publicly available databases which can be employed for both algorithm training and testing with common benchmarks. Some progress in that direction has been seen recently, specially by virtue of organizations that promote segmentation challenges, but databases are still limited to specific anatomical regions.

To summarize, in the authors' view, vessel segmentation will rapidly evolve in the direction of advanced deep-learning approaches as soon as large and labeled datasets will be publicly available. Indeed, such approaches have already drawn the attention of the computer vision community in nonmedical research fields, where large annotated datasets are already available. However, it is worth noting that collecting medical datasets big enough to encode the intra- and interpatient variability needed to justify the use of deep-learning and prevent overfitting is not trivial. This is particularly true if one considers the high variability linked to pathological tissues and the efforts needed to perform manual labeling. In the authors' opinion, this is the main reason that is slowing down the development of deep-learning algorithms for vessel segmentation. At the same time, exploiting the generalization power of deep-learning will surely help the medical computer vision community in reducing the gap between the vessel segmentation research and its use in actual clinical practice.

To conclude, this review also introduced the theoretical background of the most innovative and effective segmentation methods found in the literature, which were summarized with the support of tables reporting performance measures, datasets used and anatomical regions of interest. Pros and cons of each method were highlighted, including metrics reported by the respective authors for the specific applications. This can help developers get a better picture of the options and methods available, leading to a speed-up on the development and enhancement of methods for blood vessel segmentation.

\section{NOMENCLATURE}

- 2D: Bidimensional

- 3D: Tridimensional

- 3DRA: Tridimensional Rotation Angiography

- Acc: Accuracy

- $A U R O C$ : Area Under the Receiver Operating Characteristic Curve

- $A U P R C$ : Area Under the Precision-Recall Curve

- CNN: Convolutional Neural Network

- CRF: Conditional Random Field

- CT : Computed Tomography

- CTA : Computed Tomography Angiography

- DSA: Digital Subtraction Angiography

- DSC: Dice Similarity Coefficient

- EM: Expectation-Maximization

- FCCRF: Fully Connected Markov Random Field
- FMM: Fast Marching Method

- FN: False Negative

- FP: False Positive

- FPrate: False Positive rate

- FCN: Fully Convolutional Networks

- GF: Gabor Filter

- GPU: Graphic Processor Unit

- GS: Gold Standard

- GVF: Gradient Vector Flow

- $H$ : Hessian matrix

- HD: Hausdorff distance

- IR: Infrared

- $\kappa$ : Cohen's $\kappa$ coefficient

- LNND: Lattice Neural Network with Dendritic

- LS: Level Set

- $M C C$ : Matthews Correlation Coefficient

- $M$ : Metric tensor for minimum cost path

- MCP: Minimum Cost Path

- MF: Matched Filter

- MHTT: Multiple Hypothesis Template Tracking

- MIP: Maximum Intensity Projection

- MRA: Magnetic Resonance Angiography

- MRF: Markov Random Field

- MRI: Magnetic Resonance Imaging

- NBI: Narrow Band Imaging

- $N P V$ : Negative Predictive Value

- OCT: Optical Coherence Tomography

- $O F$ : Overlap until first error

- OOF: Optimal Oriented Flux

- OT: Overlap with the clinically relevant part of the vessel

- $O V$ : Overlap

- PBT: Probabilistic Boosting Tree

- PF: Particle Filtering

- PPV: Positive Predictive Value

- PSO: Particle Swarm Optimization

- RACAL: RAdius-based Clustering ALgorithm

- RANSAC: RANdom SAmple Consensus

- RF: Random Forest

- $R O C$ : Receiver Operating Characteristic

- Se: Sensitivity

- Sp: Specificity

- STAPLE: Simultaneous Truth And Performance Level Estimation

- SVM: Support Vector Machine

- $T N$ : True Negative

- TP: True Positive

- US: Ultrasound

\section{REFERENCES}

[1] P. Carmeliet and R. K. Jain, "Angiogenesis in cancer and other diseases," Nature, vol. 407, no. 6801, pp. 249-257, 2000.

[2] P. A. Campochiaro, "Molecular pathogenesis of retinal and choroidal vascular diseases," Progress in Retinal and Eye Research, 2015.

[3] E. De Momi, C. Caborni, F. Cardinale, G. Casaceli, L. Castana, M. Cossu, R. Mai, F. Gozzo, S. Francione, L. Tassi et al., "Multi-trajectories automatic planner for StereoElectroEncephaloGraphy (SEEG)," International Journal of Computer Assisted Radiology and Surgery, vol. 9, no. 6, pp. 1087-1097, 2014. 
[4] C. Essert, S. Fernandez-Vidal, A. Capobianco, C. Haegelen, C. Karachi, E. Bardinet, M. Marchal, and P. Jannin, "Statistical study of parameters for deep brain stimulation automatic preoperative planning of electrodes trajectories," International Journal of Computer Assisted Radiology and Surgery, vol. 10, no. 12, pp. 1973-1983, 2015.

[5] E. De Momi, C. Caborni, F. Cardinale, L. Castana, G. Casaceli, M. Cossu, L. Antiga, and G. Ferrigno, "Automatic trajectory planner for StereoElectroEncephaloGraphy procedures: a retrospective study," Transactions on Biomedical Engineering, vol. 60, no. 4, pp. 986-993, 2013.

[6] C. Faria, O. Sadowsky, E. Bicho, G. Ferrigno, L. Joskowicz, M. Shoham, R. Vivanti, and E. De Momi, "Validation of a stereo camera system to quantify brain deformation due to breathing and pulsatility," Medical Physics, vol. 41, no. 11, p. 113502, 2014.

[7] C. Piazza, F. Del Bon, G. Peretti, and P. Nicolai, "Narrow band imaging in endoscopic evaluation of the larynx," Current Opinion in Otolaryngology \& Head and Neck Surgery, vol. 20, no. 6, pp. 472-476, 2012.

[8] F. Cardinale, G. Pero, L. Quilici, M. Piano, P. Colombo, A. Moscato, L. Castana, G. Casaceli, D. Fuschillo, L. Gennari et al., "Cerebral angiography for multimodal surgical planning in epilepsy surgery: description of a new three-dimensional technique and literature review," World Neurosurgery, 2015.

[9] M. V. Schaverien and S. J. McCulley, "Contrast-enhanced magnetic resonance angiography for preoperative imaging in DIEP flap breast reconstruction," in Breast Reconstruction. Springer, 2016, pp. 163170.

[10] M. Hernández-Pérez, J. Puig, G. Blasco, N. P. de la Ossa, L. Dorado, A. Dávalos, and J. Munuera, "Dynamic magnetic resonance angiography provides collateral circulation and hemodynamic information in acute ischemic stroke," Stroke, vol. 47, no. 2, pp. 531-534, 2016.

[11] C. E. Rochitte, R. T. George, M. Y. Chen, A. Arbab-Zadeh, M. Dewey, J. M. Miller, H. Niinuma, K. Yoshioka, K. Kitagawa, S. Nakamori et al., "Computed tomography angiography and perfusion to assess coronary artery stenosis causing perfusion defects by single photon emission computed tomography: the CORE320 study," European Heart Journal, vol. 35, no. 17, pp. 1120-1130, 2014.

[12] M. M. Fraz, P. Remagnino, A. Hoppe, B. Uyyanonvara, A. R. Rudnicka, C. G. Owen, and S. A. Barman, "Blood vessel segmentation methodologies in retinal images-a survey," Computer Methods and Programs in Biomedicine, vol. 108, no. 1, pp. 407-433, 2012.

[13] C. L. Srinidhi, P. Aparna, and J. Rajan, "Recent advancements in retinal vessel segmentation," Journal of Medical Systems, vol. 41, no. 4, p. 70, 2017.

[14] C. Kirbas and F. Quek, "A review of vessel extraction techniques and algorithms," ACM Computing Surveys, vol. 36, no. 2, pp. 81-121, 2004.

[15] N. Singh and L. Kaur, "A survey on blood vessel segmentation methods in retinal images," in Electronic Design, Computer Networks \& Automated Verification, 2015 International Conference on. IEEE, 2015, pp. 23-28.

[16] K. Bühler, P. Felkel, and A. La Cruz, "Geometric methods for vessel visualization and quantification-a survey," in Geometric Modeling for Scientific Visualization. Springer, 2004, pp. 399-419.

[17] J. S. Suri, K. Liu, L. Reden, and S. Laxminarayan, "A review on MR vascular image processing: skeleton versus nonskeleton approaches: part II." Transactions on Information Technology in Biomedicine, vol. 6, no. 4, pp. 338-350, 2002.

[18] F. Molinari, G. Zeng, and J. S. Suri, "A state of the art review on intima: media thickness (imt) measurement and wall segmentation techniques for carotid ultrasound," Computer Methods and Programs in Biomedicine, vol. 100, no. 3, pp. 201-221, 2010.

[19] D. Lesage, E. D. Angelini, I. Bloch, and G. Funka-Lea, "A review of 3D vessel lumen segmentation techniques: Models, features and extraction schemes," Medical Image Analysis, vol. 13, no. 6, pp. 819-845, 2009.

[20] X. Feng, W. Xing-ce, Z. Ming-quan, W. Zhongke, and L. Xin-yu, "Segmentation algorithm of brain vessel image based on SEM statistical mixture model," in International Conference on Fuzzy Systems and Knowledge Discovery, vol. 4. IEEE, 2010, pp. 1830-1833.

[21] M. S. Hassouna, A. A. Farag, S. Hushek, and T. Moriarty, "Cerebrovascular segmentation from TOF using stochastic models," Medical Image Analysis, vol. 10, no. 1, pp. 2-18, 2006.

[22] D. A. Oliveira, R. Q. Feitosa, and M. M. Correia, "Segmentation of liver, its vessels and lesions from CT images for surgical planning," Biomed Eng Online, vol. 10, p. 30, 2011.

[23] E. Goceri, Z. K. Shah, and M. N. Gurcan, "Vessel segmentation from abdominal magnetic resonance images: adaptive and reconstructive approach," International journal for numerical methods in biomedical engineering, vol. 33, no. 4, 2017.

[24] P. Bruyninckx, D. Loeckx, D. Vandermeulen, and P. Suetens, "Segmentation of liver portal veins by global optimization," in SPIE Medical Imaging. International Society for Optics and Photonics, 2010, pp. $76241 Z-76241 Z$.

[25] P. Bruyninckx, D. Loeckx, D. Vandermeulen, and P. Suetens, "Segmentation of lung vessel trees by global optimization," in SPIE Medical Imaging, vol. 7259, 2009, p. 725912.

[26] A. H. Asad, A. T. Azar, and A. E. Hassanien, "A new heuristic function of ant colony system for retinal vessel segmentation," in Medical Imaging: Concepts, Methodologies, Tools, and Applications. IGI Global, 2017, pp. 2063-2081.

[27] T. Mapayi, J.-R. Tapamo, and S. Viriri, "Retinal vessel segmentation: a comparative study of fuzzy C-means and sum entropy information on phase congruency," International Journal of Advanced Robotic Systems, vol. 12 , no. 9 , p. 133,2015

[28] K. Sreejini and V. Govindan, "Improved multiscale matched filter for retina vessel segmentation using PSO algorithm," Egyptian Informatics Journal, vol. 16, no. 3, pp. 253-260, 2015.

[29] M. G. Cinsdikici and D. Aydın, "Detection of blood vessels in ophthalmoscope images using MF/ant (matched filter/ant colony) algorithm," Computer Methods and Programs in Biomedicine, vol. 96, no. 2, pp. 85-95, 2009.

[30] M. Al-Rawi and H. Karajeh, "Genetic algorithm matched filter optimization for automated detection of blood vessels from digital retinal images," Computer Methods and Programs in Biomedicine, vol. 87, no. 3, pp. 248-253, 2007.

[31] S. Hanaoka, Y. Nomura, M. Nemoto, S. Miki, T. Yoshikawa, N. Hayashi, K. Ohtomo, Y. Masutani, and A. Shimizu, "HoTPiG: A novel geometrical feature for vessel morphometry and its application to cerebral aneurysm detection," in International Conference on Medical Image Computing and Computer-Assisted Intervention. Springer, 2015, pp. 103-110.

[32] A. Sironi, V. Lepetit, and P. Fua, "Multiscale centerline detection by learning a scale-space distance transform," in International Conference on Computer Vision and Pattern Recognition. IEEE, 2014, pp. 26972704.

[33] J. Merkow, A. Marsden, D. Kriegman, and Z. Tu, "Dense volumeto-volume vascular boundary detection," in International Conference on Medical Image Computing and Computer-Assisted Intervention. Springer, 2016, pp. 371-379.

[34] S. Sankaran, M. Schaap, S. C. Hunley, J. K. Min, C. A. Taylor, and L. Grady, "Hale: Healthy area of lumen estimation for vessel stenosis quantification," in International Conference on Medical Image Computing and Computer-Assisted Intervention. Springer, 2016, pp. 380-387.

[35] M. Schaap, T. van Walsum, L. Neefjes, C. Metz, E. Capuano, M. de Bruijne, and W. Niessen, "Robust shape regression for supervised vessel segmentation and its application to coronary segmentation in CTA," Transactions on Medical Imaging, vol. 30, no. 11, pp. 19741986, 2011.

[36] Y. Zheng, M. Loziczonek, B. Georgescu, S. K. Zhou, F. Vega-Higuera, and D. Comaniciu, "Machine learning based vesselness measurement for coronary artery segmentation in cardiac CT volumes," in SPIE Medical Imaging, vol. 7962, 2011, pp. 79621K-1.

[37] R. Nekovei and Y. Sun, "Back-propagation network and its configuration for blood vessel detection in angiograms," Transactions on Neural Networks, vol. 6, no. 1, pp. 64-72, 1995.

[38] E. Smistad and L. Løvstakken, "Vessel detection in ultrasound images using deep convolutional neural networks," in International Workshop on Large-Scale Annotation of Biomedical Data and Expert Label Synthesis. Springer, 2016, pp. 30-38.

[39] P. Chu, Y. Pang, E. Cheng, Y. Zhu, Y. Zheng, and H. Ling, "Structureaware rank-1 tensor approximation for curvilinear structure tracking using learned hierarchical features," in International Conference on Medical Image Computing and Computer-Assisted Intervention. Springer, 2016, pp. 413-421.

[40] J. I. Orlando, E. Prokofyeva, and M. B. Blaschko, "A discriminatively trained fully connected conditional random field model for blood vessel segmentation in fundus images," Transactions on Biomedical Engineering, vol. 64, no. 1, pp. 16-27, 2017.

[41] A. Dasgupta and S. Singh, "A fully convolutional neural network based structured prediction approach towards the retinal vessel segmentation," in International Symposium on Biomedical Imaging. IEEE, 2017, pp. 248-251. 
[42] J. Mo and L. Zhang, "Multi-level deep supervised networks for retinal vessel segmentation," International Journal of Computer Assisted Radiology and Surgery, pp. 1-13, 2017.

[43] A. Lahiri, K. Ayush, P. K. Biswas, and P. Mitra, "Generative adversarial learning for reducing manual annotation in semantic segmentation on large scale miscroscopy images: Automated vessel segmentation in retinal fundus image as test case," in Conference on Computer Vision and Pattern Recognition Workshops, 2017, pp. 42-48.

[44] R. Annunziata, A. Kheirkhah, S. Aggarwal, P. Hamrah, and E. Trucco, "A fully automated tortuosity quantification system with application to corneal nerve fibres in confocal microscopy images," Medical Image Analysis, vol. 32, pp. 216-232, 2016.

[45] H. Fu, Y. Xu, S. Lin, D. W. K. Wong, and J. Liu, "DeepVessel: Retinal vessel segmentation via deep learning and conditional random field," in International Conference on Medical Image Computing and ComputerAssisted Intervention. Springer, 2016, pp. 132-139.

[46] Y. Luo, L. Yang, L. Wang, and H. Cheng, "Efficient CNN-CRF network for retinal image segmentation," in International Conference on Cognitive Systems and Signal Processing. Springer, 2016, pp. $157-165$

[47] P. Liskowski and K. Krawiec, "Segmenting retinal blood vessels with deep neural networks," Transactions on Medical Imaging, vol. 35 , no. 11, pp. 2369-2380, 2016.

[48] Q. Li, B. Feng, L. Xie, P. Liang, H. Zhang, and T. Wang, "A crossmodality learning approach for vessel segmentation in retinal images," Transactions on Medical Imaging, vol. 35, no. 1, pp. 109-118, 2016.

[49] M. Javidi, H.-R. Pourreza, and A. Harati, "Vessel segmentation and microaneurysm detection using discriminative dictionary learning and sparse representation," Computer Methods and Programs in Biomedicine, 2016

[50] K.-K. Maninis, J. Pont-Tuset, P. Arbeláez, and L. Van Gool, "Deep retinal image understanding," in International Conference on Medical Image Computing and Computer-Assisted Intervention. Springer, 2016, pp. 140-148.

[51] P. Prentašić, M. Heisler, Z. Mammo, S. Lee, A. Merkur, E. Navajas, M. F. Beg, M. Šarunić, and S. Lončarić, "Segmentation of the foveal microvasculature using deep learning networks," Journal of Biomedical Optics, vol. 21, no. 7, pp. $075008-075008,2016$.

[52] A. Wu, Z. Xu, M. Gao, M. Buty, and D. J. Mollura, "Deep vessel tracking: A generalized probabilistic approach via deep learning," in International Symposium on Biomedical Imaging. IEEE, 2016, pp. $1363-1367$.

[53] R. Annunziata, A. Kheirkhah, P. Hamrah, and E. Trucco, "Scale and curvature invariant ridge detector for tortuous and fragmented structures," in International Conference on Medical Image Computing and Computer-Assisted Intervention. Springer, 2015, pp. 588-595.

[54] R. Annunziata, A. Kheirkhah, P. Hamrah, and E. Trucco, "Boosting hand-crafted features for curvilinear structure segmentation by learning context filters," in International Conference on Medical Image Computing and Computer-Assisted Intervention. Springer, 2015, pp. 596-603.

[55] R. Vega, G. Sanchez-Ante, L. E. Falcon-Morales, H. Sossa, and E. Guevara, "Retinal vessel extraction using lattice neural networks with dendritic processing," Computers in Biology and Medicine, vol. 58, pp. 20-30, 2015.

[56] S. Wang, Y. Yin, G. Cao, B. Wei, Y. Zheng, and G. Yang, "Hierarchical retinal blood vessel segmentation based on feature and ensemble learning," Neurocomputing, vol. 149, pp. 708-717, 2015.

[57] M. M. Fraz, A. R. Rudnicka, C. G. Owen, and S. A. Barman, "Delineation of blood vessels in pediatric retinal images using decision trees-based ensemble classification," International Journal of Computer Assisted Radiology and Surgery, vol. 9, no. 5, pp. 795-811, 2014.

[58] Y. Ganin and V. Lempitsky, " $\mathrm{N}^{\wedge}$ 4-Fields: Neural Network Nearest Neighbor Fields for Image Transforms," in Asian Conference on Computer Vision. Springer, 2014, pp. 536-551.

[59] J. I. Orlando and M. Blaschko, "Learning fully-connected CRFs for blood vessel segmentation in retinal images," in Medical Image Computing and Computer-Assisted Intervention. Springer, 2014, pp. 634-641.

[60] C. Becker, R. Rigamonti, V. Lepetit, and P. Fua, "Supervised feature learning for curvilinear structure segmentation," in International Conference on Medical Image Computing and Computer-Assisted Intervention. Springer, 2013, pp. 526-533.

[61] P. Rodrigues, P. Guimarães, T. Santos, S. Simão, T. Miranda, P. Serranho, and R. Bernardes, "Two-dimensional segmentation of the retinal vascular network from optical coherence tomography," Journal of Biomedical Optics, vol. 18, no. 12, pp. 126011-126011, 2013.
[62] M. M. Fraz, P. Remagnino, A. Hoppe, B. Uyyanonvara, A. R. Rudnicka, C. G. Owen, and S. A. Barman, "An ensemble classificationbased approach applied to retinal blood vessel segmentation," Transactions on Biomedical Engineering, vol. 59, no. 9, pp. 2538-2548, 2012.

[63] B. Zhang, F. Karray, Q. Li, and L. Zhang, "Sparse representation classifier for microaneurysm detection and retinal blood vessel extraction," Information Sciences, vol. 200, pp. 78-90, 2012.

[64] D. Marín, A. Aquino, M. E. Gegúndez-Arias, and J. M. Bravo, "A new supervised method for blood vessel segmentation in retinal images by using gray-level and moment invariants-based features," Transactions on Medical Imaging, vol. 30, no. 1, pp. 146-158, 2011.

[65] C. A. Lupascu, D. Tegolo, and E. Trucco, "FABC: retinal vessel segmentation using AdaBoost," Transactions on Information Technology in Biomedicine, vol. 14, no. 5, pp. 1267-1274, 2010.

[66] N. Salem, S. Salem, and A. Nandi, "Segmentation of retinal blood vessels based on analysis of the Hessian matrix and clustering algorithm," in European Signal Processing Conference, 2007, pp. 428-432.

[67] J. V. Soares, J. J. Leandro, R. M. Cesar, H. F. Jelinek, and M. J. Cree, "Retinal vessel segmentation using the 2-D Gabor wavelet and supervised classification," Transactions on Medical Imaging, vol. 25, no. 9, pp. 1214-1222, 2006.

[68] J. Staal, M. D. Abràmoff, M. Niemeijer, M. A. Viergever, and B. van Ginneken, "Ridge-based vessel segmentation in color images of the retina," Transactions on Medical Imaging, vol. 23, no. 4, pp. 501-509, 2004

[69] S.-H. Lee and S. Lee, "Adaptive Kalman snake for semi-autonomous 3D vessel tracking," Computer Methods and Programs in Biomedicine, vol. 122 , no. 1, pp. 56-75, 2015.

[70] L. F. Valencia, J. Montagnat, and M. Orkisz, "3D models for vascular lumen segmentation in MRA images and for artery-stenting simulation," IRBM, vol. 28, no. 2, pp. 65-71, 2007.

[71] M. W. Law and A. C. Chung, "An oriented flux symmetry based active contour model for three dimensional vessel segmentation," in European Conference on Computer Vision. Springer, 2010, pp. 720-734.

[72] R. Moreno, C. Wang, and Ö. Smedby, "Vessel wall segmentation using implicit models and total curvature penalizers," in Image Analysis. Springer, 2013, pp. 299-308.

[73] C. Wang, R. Moreno, and Ö. Smedby, "Vessel segmentation using implicit model-guided level sets," in MICCAI Workshop" 3D Cardiovascular Imaging", Nice France, 1st of October 2012., 2012.

[74] Y. Cheng, X. Hu, J. Wang, Y. Wang, and S. Tamura, "Accurate vessel segmentation with constrained B-snake," Transaction on Image Processing, vol. 24, no. 8, pp. 2440-2455, 2015.

[75] X. Zhu, Z. Xue, X. Gao, Y. Zhu, and S. T. Wong, "Voles: Vascularityoriented level set algorithm for pulmonary vessel segmentation in image guided intervention therapy," in International Symposium on Biomedical Imaging. IEEE, 2009, pp. 1247-1250.

[76] J. Zhang, Z. Tang, W. Gui, and J. Liu, "Retinal vessel image segmentation based on correlational open active contours model," in Chinese Automation Congress. IEEE, 2015, pp. 993-998.

[77] K. A. Patwardhan, Y. Yu, S. Gupta, A. Dentinger, and D. Mills, " $4 d$ vessel segmentation and tracking in ultrasound," in Image Processing. Proceeding of the 19th International Conference on. IEEE, 2012, pp. 2317-2320.

[78] A. Klepaczko, P. Szczypiński, A. Deistung, J. R. Reichenbach, and A. Materka, "Simulation of MR angiography imaging for validation of cerebral arteries segmentation algorithms," Computer Methods and Programs in Biomedicine, vol. 137, pp. 293-309, 2016.

[79] Y. Tian, Q. Chen, W. Wang, Y. Peng, Q. Wang, F. Duan, Z. Wu, and M. Zhou, "A Vessel Active Contour Model for Vascular Segmentation," BioMed Research International, 2014.

[80] M. W. Law and A. C. Chung, "Weighted local variance-based edge detection and its application to vascular segmentation in magnetic resonance angiography," Transactions on Medical Imaging, vol. 26, no. 9, pp. 1224-1241, 2007.

[81] L. Wang, L. He, A. Mishra, and C. Li, "Active contours driven by local Gaussian distribution fitting energy," Signal Processing, vol. 89, no. 12, pp. 2435-2447, 2009.

[82] Y. Liang, F. Wang, D. Treanor, D. Magee, G. Teodoro, Y. Zhu, and J. Kong, "A 3D primary vessel reconstruction framework with serial microscopy images," in International Conference on Medical Image Computing and Computer-Assisted Intervention. Springer, 2015, pp. 251-259.

[83] Y. Zhao, Y. Liu, X. Wu, S. P. Harding, and Y. Zheng, "Retinal vessel segmentation: An efficient graph cut approach with retinex and local phase," PloS One, vol. 10, no. 4, p. e0122332, 2015. 
[84] Y. Zhao, L. Rada, K. Chen, S. P. Harding, and Y. Zheng, "Automated vessel segmentation using infinite perimeter active contour model with hybrid region information with application to retinal images,' Transactions on Medical Imaging, vol. 34, no. 9, pp. 1797-1807, 2015.

[85] L. Wang, H. Zhang, K. He, Y. Chang, and X. Yang, "Active Contours Driven by Multi-Feature Gaussian Distribution Fitting Energy with Application to Vessel Segmentation," PloS One, vol. 10, no. 11, p. $\mathrm{e} 0143105,2015$.

[86] Z. Xiao, M. Adel, and S. Bourennane, "Bayesian method with spatial constraint for retinal vessel segmentation," Computational and Mathematical Methods in Medicine, vol. 2013, 2013.

[87] W. K. Law and A. C. Chung, "Segmentation of vessels using weighted local variances and an active contour model," in Conference on Computer Vision and Pattern Recognition Workshop. IEEE, 2006, pp. 83-83.

[88] D. Robben, E. Türetken, S. Sunaert, V. Thijs, G. Wilms, P. Fua, F. Maes, and P. Suetens, "Simultaneous segmentation and anatomical labeling of the cerebral vasculature," Medical Image Analysis, vol. 32, pp. 201-215, 2016.

[89] M. Rempfler, M. Schneider, G. D. Ielacqua, X. Xiao, S. R. Stock, J. Klohs, G. Székely, B. Andres, and B. H. Menze, "Reconstructing cerebrovascular networks under local physiological constraints by integer programming," Medical Image Analysis, vol. 25, no. 1, pp. 86-94, 2015.

[90] A. Yureidini, E. Kerrien, and S. Cotin, "Robust RANSAC-based blood vessel segmentation," in SPIE Medical Imaging. International Society for Optics and Photonics, 2012, pp. $83141 \mathrm{M}-83141 \mathrm{M}$.

[91] S. Cetin and G. Unal, "A higher-order tensor vessel tractography for segmentation of vascular structures," Transactions on Medical Imaging, vol. 34 , no. 10 , pp. $2172-2185,2015$.

[92] S. Cetin, A. Demir, A. Yezzi, M. Degertekin, and G. Unal, "Vessel tractography using an intensity based tensor model with branch detection," Transactions on Medical Imaging, vol. 32, no. 2, pp. 348-363, 2013

[93] H. Shim, D. Kwon, I. D. Yun, and S. U. Lee, "Robust segmentation of cerebral arterial segments by a sequential Monte Carlo method: Particle filtering," Computer Methods and Programs in Niomedicine, vol. 84, no. 2, pp. 135-145, 2006.

[94] K. M. Cherry, B. Peplinski, L. Kim, S. Wang, L. Lu, W. Zhang, J. Liu, Z. Wei, and R. M. Summers, "Sequential Monte Carlo tracking of the marginal artery by multiple cue fusion and random forest regression," Medical Image Analysis, vol. 19, no. 1, pp. 164-175, 2015.

[95] S. Y. Shin, S. Lee, K. J. Noh, I. D. Yun, and K. M. Lee, "Extraction of coronary vessels in fluoroscopic X-Ray sequences using vessel correspondence optimization," in International Conference on Medical Image Computing and Computer-Assisted Intervention. Springer, 2016, pp. 308-316.

[96] J. F. Carrillo, M. H. Hoyos, E. E. Dávila, and M. Orkisz, "Recursive tracking of vascular tree axes in 3D medical images," International Journal of Computer Assisted Radiology and Surgery, vol. 1, no. 6, pp. 331-339, 2007.

[97] A. Amir-Khalili, G. Hamarneh, and R. Abugharbieh, "Automatic vessel segmentation from pulsatile radial distension," in International Conference on Medical Image Computing and Computer-Assisted Intervention. Springer, 2015, pp. 403-410.

[98] F. Benmansour and L. D. Cohen, "Tubular structure segmentation based on minimal path method and anisotropic enhancement," International Journal of Computer Vision, vol. 92, no. 2, pp. 192-210, 2011.

[99] A. Biesdorf, S. Wörz, H. von Tengg-Kobligk, K. Rohr, and C. Schnörr, "3D segmentation of vessels by incremental implicit polynomial fitting and convex optimization," in International Symposium on Biomedical Imaging. IEEE, 2015, pp. 1540-1543.

[100] F. Lugauer, Y. Zheng, J. Hornegger, and B. M. Kelm, "Precise lumen segmentation in coronary computed tomography angiography," in International MICCAI Workshop on Medical Computer Vision. Springer, 2014, pp. 137-147.

[101] H. Tang, T. van Walsum, R. S. van Onkelen, R. Hameeteman, S. Klein, M. Schaap, F. L. Tori, Q. J. van den Bouwhuijsen, J. C. Witteman, A. van der Lugt et al., "Semiautomatic carotid lumen segmentation for quantification of lumen geometry in multispectral MRI," Medical Image Analysis, vol. 16, no. 6, pp. 1202-1215, 2012.

[102] X. Wang, T. Heimann, P. Lo, M. Sumkauskaite, M. Puderbach, M. de Bruijne, H. Meinzer, and I. Wegner, "Statistical tracking of treelike tubular structures with efficient branching detection in 3D medical image data," Physics in Medicine and Biology, vol. 57, no. 16, p. 5325, 2012.
[103] O. Friman, M. Hindennach, C. Kühnel, and H.-O. Peitgen, "Multiple hypothesis template tracking of small 3D vessel structures," Medical Image Analysis, vol. 14, no. 2, pp. 160-171, 2010.

[104] H. Li, A. Yezzi, and L. Cohen, "3D multi-branch tubular surface and centerline extraction with 4D iterative key points," in International Conference on Medical Image Computing and Computer-Assisted Intervention. Springer, 2009, pp. 1042-1050.

[105] O. Wink, A. F. Frangi, B. Verdonck, M. A. Viergever, and W. J. Niessen, "3D MRA coronary axis determination using a minimum cost path approach," Magnetic Resonance in Medicine, vol. 47, no. 6, pp. $1169-1175,2002$.

[106] Y.-z. Zeng, Y.-q. Zhao, P. Tang, M. Liao, Y.-x. Liang, S.-h. Liao, and B.-j. Zou, "Liver vessel segmentation and identification based on oriented flux symmetry and graph cuts," Computer Methods and Programs in Biomedicine, vol. 150, pp. 31-39, 2017.

[107] C. Bauer, T. Pock, E. Sorantin, H. Bischof, and R. Beichel, "Segmentation of interwoven 3D tubular tree structures utilizing shape priors and graph cuts," Medical Image Analysis, vol. 14, no. 2, pp. 172-184, 2010 .

[108] A. Amir-Khalili, G. Hamarneh, J.-M. Peyrat, J. Abinahed, O. AlAlao, A. Al-Ansari, and R. Abugharbieh, "Automatic segmentation of occluded vasculature via pulsatile motion analysis in endoscopic robotassisted partial nephrectomy video," Medical Image Analysis, vol. 25, no. 1, pp. 103-110, 2015.

[109] D. Chen, J.-M. Mirebeau, and L. D. Cohen, "Vessel tree extraction using radius-lifted keypoints searching scheme and anisotropic fast marching method," Journal of Algorithms \& Computational Technology, p. 1748301816656289, 2016.

[110] D. Chen, L. D. Cohen, and J.-M. Mirebeau, "Vessel extraction using anisotropic minimal paths and path score," in International Conference on Image Processing. IEEE, 2014, pp. 1570-1574.

[111] A. Bhuiyan, R. Kawasaki, E. Lamoureux, K. Ramamohanarao, and T. Y. Wong, "Retinal artery-vein caliber grading using color fundus imaging," Computer Methods and Programs in Biomedicine, vol. 111, no. 1, pp. 104-114, 2013.

[112] W. Liao, K. Rohr, and S. Wörz, "Globally optimal curvatureregularized fast marching for vessel segmentation," in International Conference on Medical Image Computing and Computer-Assisted Intervention. Springer, 2013, pp. 550-557.

[113] Y. Rouchdy and L. D. Cohen, "Geodesic voting for the automatic extraction of tree structures. Methods and applications," Computer Vision and Image Understanding, vol. 117, no. 10, pp. 1453-1467, 2013.

[114] J. Stuhmer, P. Schroder, and D. Cremers, "Tree shape priors with connectivity constraints using convex relaxation on general graphs," in International Conference on Computer Vision, pp. 2336-2343.

[115] E. Turetken, F. Benmansour, B. Andres, H. Pfister, and P. Fua, "Reconstructing loopy curvilinear structures using integer programming," in Proceedings of the IEEE Conference on Computer Vision and Pattern Recognition, 2013, pp. 1822-1829.

[116] W. Liao, S. Wörz, and K. Rohr, "Globally minimal path method using dynamic speed functions based on progressive wave propagation," in Asian Conference on Computer Vision. Springer, 2012, pp. 25-37.

[117] V. Kaul, A. Yezzi, and Y. Tsai, "Detecting curves with unknown endpoints and arbitrary topology using minimal paths," Transactions on Pattern Analysis and Machine Intelligence, vol. 34, no. 10, pp. 1952-1965, 2012.

[118] K. K. Delibasis, A. I. Kechriniotis, C. Tsonos, and N. Assimakis, "Automatic model-based tracing algorithm for vessel segmentation and diameter estimation," Computer Methods and Programs in Biomedicine, vol. 100, no. 2, pp. 108-122, 2010.

[119] D. Breitenreicher, M. Sofka, S. Britzen, and S. K. Zhou, "Hierarchical discriminative framework for detecting tubular structures in 3D images," in International Conference on Information Processing in Medical Imaging. Springer, 2013, pp. 328-339.

[120] F. Benmansour and L. D. Cohen, "Fast object segmentation by growing minimal paths from a single point on 2D or 3D images," Journal of Mathematical Imaging and Vision, vol. 33, no. 2, pp. 209-221, 2009.

[121] O. Wink, W. J. Niessen, and M. A. Viergever, "Multiscale vesse tracking," Transactions on Medical Imaging, vol. 23, no. 1, pp. 130133, 2004.

[122] S. Krig, "Image Pre-Processing," in Computer Vision Metrics. Springer, 2014, pp. 39-83.

[123] S. Chaudhuri, S. Chatterjee, N. Katz, M. Nelson, and M. Goldbaum, "Detection of blood vessels in retinal images using two-dimensional matched filters," Transactions on Medical Imaging, vol. 8, no. 3, pp. 263-269, 1989. 
[124] A. F. Frangi, W. J. Niessen, K. L. Vincken, and M. A. Viergever "Multiscale vessel enhancement filtering," in International Conference on Medical Image Computing and Computer-Assisted Interventation. Springer, 1998, pp. 130-137.

[125] K. Krissian, G. Malandain, and N. Ayache, "Directional anisotropic diffusion applied to segmentation of vessels in 3D images," in International Conference on Scale-Space Theories in Computer Vision. Springer, 1997, pp. 345-348.

[126] L. P. Cordella, P. Foggia, C. Sansone, F. Tortorella, and M. Vento, "Reliability parameters to improve combination strategies in multiexpert systems," Pattern Analysis \& Applications, vol. 2, no. 3, pp. 205-214, 1999.

[127] S. K. Warfield, K. H. Zou, and W. M. Wells, "Simultaneous truth and performance level estimation (STAPLE): an algorithm for the validation of image segmentation," Transactions on Medical Imaging, vol. 23, no. 7, pp. 903-921, 2004

[128] D. Faraggi and B. Reiser, "Estimation of the area under the ROC curve," Statistics in Medicine, vol. 21, no. 20, pp. 3093-3106, 2002.

[129] D. M. Powers, "Evaluation: from precision, recall and F-measure to ROC, informedness, markedness and correlation," 2011.

[130] J. Cohen, "Weighted kappa: Nominal scale agreement provision for scaled disagreement or partial credit." Psychological Bulletin, vol. 70, no. 4, p. 213, 1968.

[131] L. R. Dice, "Measures of the amount of ecologic association between species," Ecology, vol. 26, no. 3, pp. 297-302, 1945.

[132] M. E. Gegúndez-Arias, A. Aquino, J. M. Bravo, and D. Marin, "A function for quality evaluation of retinal vessel segmentations," Transactions on Medical Imaging, vol. 31, no. 2, pp. 231-239, 2012.

[133] R. M. Haralick, S. R. Sternberg, and X. Zhuang, "Image analysis using mathematical morphology," Transactions on Pattern Analysis and Machine Intelligence, no. 4, pp. 532-550, 1987.

[134] C. Metz, M. Schaap, T. van Walsum, A. van der Giessen, A. Weustink, N. Mollet, G. Krestin, and W. Niessen, "3D segmentation in the clinic: A grand challenge II-coronary artery tracking," Insight Journal, vol. 1, no. 5, p. 6, 2008.

[135] T. van Walsum, M. Schaap, C. Metz, A. van der Giessen, and W. Niessen, "Averaging centerlines: mean shift on paths," International Conference on Medical Image Computing and Computer-Assisted Intervention, pp. 900-907, 2008.

[136] A. Hoover, V. Kouznetsova, and M. Goldbaum, "Locating blood vessels in retinal images by piecewise threshold probing of a matched filter response," Transactions on Medical Imaging, vol. 19, no. 3, pp. 203210, 2000.

[137] D. J. Farnell, F. Hatfield, P. Knox, M. Reakes, S. Spencer, D. Parry, and S. Harding, "Enhancement of blood vessels in digital fundus photographs via the application of multiscale line operators," Journal of the Franklin Institute, vol. 345, no. 7, pp. 748-765, 2008.

[138] C. G. Owen, A. R. Rudnicka, R. Mullen, S. A. Barman, D. Monekosso, P. H. Whincup, J. Ng, and C. Paterson, "Measuring retinal vessel tortuosity in 10-year-old children: validation of the Computer-Assisted Image Analysis of the Retina (CAIAR) program," Investigative Ophthalmology \& Visual Science, vol. 50, no. 5, pp. 2004-2010, 2009.

[139] J. Odstrcilik, R. Kolar, A. Budai, J. Hornegger, J. Jan, J. Gazarek, T. Kubena, P. Cernosek, O. Svoboda, and E. Angelopoulou, "Retinal vessel segmentation by improved matched filtering: evaluation on a new high-resolution fundus image database," IET Image Processing, vol. 7, no. 4, pp. 373-383, 2013.

[140] R. V. J. P. H. Kälviäinen and H. Uusitalo, "DIARETDB1 diabetic retinopathy database and evaluation protocol," Medical Image Understanding and Analysis, p. 61, 2007.

[141] B. Al-Diri, A. Hunter, D. Steel, M. Habib, T. Hudaib, and S. Berry, "REVIEW-a reference data set for retinal vessel profiles," in International Conference of the IEEE Engineering in Medicine and Biology Society. IEEE, 2008, pp. 2262-2265.

[142] M. Niemeijer, B. Van Ginneken, M. J. Cree, A. Mizutani, G. Quellec, C. I. Sánchez, B. Zhang, R. Hornero, M. Lamard, C. Muramatsu et al., "Retinopathy online challenge: automatic detection of microaneurysms in digital color fundus photographs," Transaction on Medical Imaging, vol. 29, no. 1, pp. 185-195, 2010.

[143] H. Kirişli, M. Schaap, C. Metz, A. Dharampal, W. B. Meijboom, S. Papadopoulou, A. Dedic, K. Nieman, M. De Graaf, M. Meijs et al., "Standardized evaluation framework for evaluating coronary artery stenosis detection, stenosis quantification and lumen segmentation algorithms in computed tomography angiography," Medical Image Analysis, vol. 17, no. 8, pp. 859-876, 2013.

[144] M. Schaap, C. T. Metz, T. van Walsum, A. G. van der Giessen, A. C. Weustink, N. R. Mollet, C. Bauer, H. Bogunović, C. Castro, X. Deng et al., "Standardized evaluation methodology and reference database for evaluating coronary artery centerline extraction algorithms," Medical Image Analysis, vol. 13, no. 5, pp. 701-714, 2009.

[145] R. D. Rudyanto, S. Kerkstra, E. M. Van Rikxoort, C. Fetita, P. Y. Brillet, C. Lefevre, W. Xue, X. Zhu, J. Liang, İ. Öksüz et al., "Comparing algorithms for automated vessel segmentation in computed tomography scans of the lung: the VESSEL12 study," Medical Image Analysis, vol. 18, no. 7, pp. 1217-1232, 2014.

[146] P. Jassi and G. Hamarneh, "Vascusynth: Vascular tree synthesis software," Insight Journal, vol. January-June, pp. 1-12, 2011.

[147] T. K. Moon, "The expectation-maximization algorithm," Signal Processing Magazine, vol. 13, no. 6, pp. 47-60, 1996.

[148] S. Z. Li, Markov random field modeling in image analysis. Springer Science \& Business Media, 2009

[149] B. S. Everitt, Finite mixture distributions. Wiley Online Library, 1981.

[150] P. Kovesi, "Image features from phase congruency," Videre: Journal of Computer Vision Research, vol. 1, no. 3, pp. 1-26, 1999.

[151] R. Annunziata, A. Kheirkhah, S. Aggarwal, B. M. Cavalcanti, P. Hamrah, and E. Trucco, "Tortuosity classification of corneal nerves images using a multiple-scale-multiple-window approach," 2014

[152] R. Rigamonti and V. Lepetit, "Accurate and efficient linear structure segmentation by leveraging ad hoc features with learned filters," International Conference on Medical Image Computing and ComputerAssisted Intervention, pp. 189-197, 2012.

[153] T. Joachims, T. Finley, and C.-N. J. Yu, "Cutting-plane training of structural SVMs," Machine Learning, vol. 77, no. 1, pp. 27-59, 2009.

[154] S. A. Salem and A. K. Nandi, "Novel clustering algorithm (RACAL) and a partial supervision strategy for classification," in Machine Learning for Signal Processing. Proceedings of the Signal Processing Society Workshop on. IEEE, 2006, pp. 313-318.

[155] Z. Tu and X. Bai, "Auto-context and its application to high-level vision tasks and 3D brain image segmentation," Transactions on Pattern Analysis and Machine Intelligence, vol. 32, no. 10, pp. 1744-1757, 2010.

[156] M. W. Law and A. C. Chung, "Three dimensional curvilinear structure detection using optimally oriented flux," in Computer Vision. Springer, 2008, pp. 368-382.

[157] D.-X. Xue, R. Zhang, H. Feng, and Y.-L. Wang, "Cnn-SVM for microvascular morphological type recognition with data augmentation," Journal of Medical and Biological Engineering, vol. 36, no. 6, pp. 755764, 2016.

[158] A. Radford, L. Metz, and S. Chintala, "Unsupervised representation learning with deep convolutional generative adversarial networks," arXiv Preprint arXiv:1511.06434, 2015.

[159] C. Xu, D. L. Pham, and J. L. Prince, "Image segmentation using deformable models," Handbook of Medical Imaging, vol. 2, pp. 129174,2000

[160] M. Kass, A. Witkin, and D. Terzopoulos, "Snakes: Active contour models," International Journal of Computer Vision, vol. 1, no. 4, pp. 321-331, 1988.

[161] P. Brigger, J. Hoeg, and M. Unser, "B-spline snakes: a flexible tool for parametric contour detection," Transactions on Image Processing, vol. 9, no. 9, pp. 1484-1496, 2000.

[162] C. Xu and J. L. Prince, "Generalized gradient vector flow external forces for active contours," Signal Processing, vol. 71, no. 2, pp. 131139, 1998.

[163] V. Caselles, F. Catté, T. Coll, and F. Dibos, "A geometric model for active contours in image processing," Numerische Mathematik, vol. 66 , no. 1, pp. 1-31, 1993

[164] R. Malladi, J. A. Sethian, and B. C. Vemuri, "Shape modeling with front propagation: A level set approach," Transaction on Pattern Analysis and Machine Intelligence, vol. 17, no. 2, pp. 158-175, 1995.

[165] A. Tannenbaum, "Three snippets of curve evolution theory in computer vision," Mathematical and Computer Modelling, vol. 24, no. 5, pp. 103-119, 1996.

[166] S. Osher and J. A. Sethian, "Fronts propagating with curvaturedependent speed: algorithms based on Hamilton-Jacobi formulations," Journal of Computational Physics, vol. 79, no. 1, pp. 12-49, 1988.

[167] V. Caselles, R. Kimmel, and G. Sapiro, "Geodesic active contours," International Journal of Computer Vision, vol. 22, no. 1, pp. 61-79, 1997.

[168] A. Yezzi Jr, S. Kichenassamy, A. Kumar, P. Olver, and A. Tannenbaum, "A geometric snake model for segmentation of medical imagery," Transactions on Medical Imaging, vol. 16, no. 2, pp. 199-209, 1997.

[169] T. Deschamps and L. D. Cohen, "Fast extraction of tubular and tree 3D surfaces with front propagation methods," in International Conference on Pattern Recognition. Proceedings, vol. 1. IEEE, 2002, pp. 731-734. 
[170] T. Deschamps, P. Schwartz, D. Trebotich, P. Colella, D. Saloner, and R. Malladi, "Vessel segmentation and blood flow simulation using level-sets and embedded boundary methods," in International Congress Series, vol. 1268. Elsevier, 2004, pp. 75-80.

[171] M. B. Milwer, L. F. Valencia, M. H. Hoyos, I. E. Magnin, and M. Orkisz, "Fast-marching contours for the segmentation of vessel lumen in CTA cross-sections," in Annual International Conference of the IEEE Engineering in Medicine and Biology Society. IEEE, 2007, pp. 791-794.

[172] M. Orkisz, L. Flórez Valencia, and M. Hernández Hoyos, "Models, algorithms and applications in vascular image segmentation," Machine Graphics and Vision, vol. 17, no. 1, pp. 5-33, 2008.

[173] J. A. Sethian, "Fast marching methods," SIAM Review, vol. 41, no. 2, pp. 199-235, 1999.

[174] D. Chopp and J. A. Sethian, "Motion by intrinsic Laplacian of curvature," Interfaces and Free Boundaries, vol. 1, no. 1, pp. 107-123, 1999.

[175] M. W. Law and A. C. Chung, "Efficient implementation for spherical flux computation and its application to vascular segmentation," Transactions on Image Processing, vol. 18, no. 3, pp. 596-612, 2009.

[176] S. Bouix, K. Siddiqi, and A. Tannenbaum, "Flux driven automatic centerline extraction," Medical Image Analysis, vol. 9, no. 3, pp. 209221, 2005.

[177] S. Bouix, K. Siddiqi, and A. Tannenbaum, "Flux driven fly throughs," in International Conference on Computer Vision and Pattern Recognition., vol. 1. IEEE, 2003, pp. I-449.

[178] M. S. Hassouna and A. A. Farag, "Robust centerline extraction framework using level sets," in Computer Society Conference on Computer Vision and Pattern Recognition, vol. 1. IEEE, 2005, pp. 458-465.

[179] A. Vasilevskiy and K. Siddiqi, "Flux maximizing geometric flows," Transactions on Pattern Analysis and Machine Intelligence, vol. 24, no. 12, pp. 1565-1578, 2002.

[180] R. Moreno and Ö. Smedby, "Gradient-based enhancement of tubular structures in medical images," Medical Image Analysis, vol. 26, no. 1, pp. 19-29, 2015.

[181] T. F. Chan and L. A. Vese, "Active contours without edges," Transaction on Image processing, vol. 10, no. 2, pp. 266-277, 2001.

[182] J. Mille and L. D. Cohen, "A local normal-based region term for active contours." in International Conference on Energy Minimization Methods in Computer Vision and Pattern Recognition. Springer, 2009, pp. $168-181$.

[183] C. Li, C.-Y. Kao, J. C. Gore, and Z. Ding, "Minimization of regionscalable fitting energy for image segmentation," Transaction on Image Processing, vol. 17, no. 10, pp. 1940-1949, 2008.

[184] G. Läthén, J. Jonasson, and M. Borga, "Blood vessel segmentation using multi-scale quadrature filtering," Pattern Recognition Letters, vol. 31, no. 8, pp. 762-767, 2010.

[185] M. Schaap, L. Neefjes, C. Metz, A. van der Giessen, A. Weustink, N. Mollet, J. Wentzel, T. van Walsum, and W. Niessen, "Coronary lumen segmentation using graph cuts and robust kernel regression," in Information Processing in Medical Imaging. Springer, 2009, pp. $528-539$.

[186] K. Hameeteman, M. Freiman, M. Zuluaga, L. Joskowicz, S. Rozie, M. Van Gils, L. Van den Borne, J. Sosna, P. Berman, N. Cohen et al., "Carotid lumen segmentation and stenosis grading challenge," The Midas Journal, 2009.

[187] D. Lesage, E. D. Angelini, I. Bloch, and G. Funka-Lea, "Design and study of flux-based features for 3D vascular tracking," in International Symposium on Biomedical Imaging. IEEE, 2009, pp. 286-289.

[188] A. Doucet, S. Godsill, and C. Andrieu, "On sequential Monte Carlo sampling methods for bayesian filtering," Statistics and Computing, vol. 10, no. 3, pp. 197-208, 2000

[189] R. C. Bolles and M. A. Fischler, "A RANSAC-Based Approach to Model Fitting and Its Application to Finding Cylinders in Range Data." in International Joint Conference on Artificial Intelligence, , vol. 1981, 1981, pp. 637-643.

[190] Y. Y. Boykov and M.-P. Jolly, "Interactive graph cuts for optimal boundary \& region segmentation of objects in nd images," in International Conference on Computer Vision, vol. 1. IEEE, 2001, pp. 105-112.

[191] M. Felsberg and G. Sommer, "The monogenic signal," Transactions on Signal Processing, vol. 49, no. 12, pp. 3136-3144, 2001.

[192] L. D. Cohen and R. Kimmel, "Global minimum for active contour models: A minimal path approach," International Journal of Computer Vision, vol. 24, no. 1, pp. 57-78, 1997.
[193] T. Deschamps and L. D. Cohen, "Fast extraction of minimal paths in 3D images and applications to virtual endoscopy," Medical Image Analysis, vol. 5, no. 4, pp. 281-299, 2001.

[194] L. D. Cohen and T. Deschamps, "Grouping connected components using minimal path techniques. Application to reconstruction of vessels in 2D and 3D images," in Computer Vision and Pattern Recognition.

[195] J. A. Sethian, "A fast marching level set method for monotonically advancing fronts," Proceedings of the National Academy of Sciences, vol. 93, no. 4, pp. 1591-1595, 1996

[196] J. N. Tsitsiklis, "Efficient algorithms for globally optimal trajectories," Transactions on Automatic Control, vol. 40, no. 9, pp. 1528-1538, 1995.

[197] S. M. Hassouna and A. A. Farag, "Multistencils fast marching methods: A highly accurate solution to the eikonal equation on cartesian domains," Transactions on Pattern Analysis and Machine Intelligence, vol. 29, no. 9, pp. 1563-1574, 2007.

[198] J. A. Sethian and A. Vladimirsky, "Fast methods for the eikonal and related Hamilton-Jacobi equations on unstructured meshes," Proceedings of the National Academy of Sciences, vol. 97, no. 11, pp. 5699-5703, 2000.

[199] H. Li and A. Yezzi, "Vessels as 4-D curves: Global minimal 4-D paths to extract 3-D tubular surfaces and centerlines," Transactions on Medical Imaging, vol. 26, no. 9, pp. 1213-1223, 2007.

[200] E. Konukoglu, M. Sermesant, O. Clatz, J.-M. Peyrat, H. Delingette, and N. Ayache, "A recursive anisotropic fast marching approach to reaction diffusion equation: Application to tumor growth modeling," in Information Processing in Medical Imaging. Springer, 2007, pp. 687-699.

[201] A. Jameson, W. Schmidt, E. Turkel et al., "Numerical solutions of the Euler equations by finite volume methods using Runge-Kutta timestepping schemes," AIAA Paper, vol. 1259, p. 1981, 1981.

[202] M. A. Gülsün and H. Tek, "Robust vessel tree modeling," in International Conference on Medical Image Computing and ComputerAssisted Intervention. Springer, 2008, pp. 602-611.

[203] H. Tang, T. Van Walsum, R. S. Van Onkelen, S. Klein, R. Hameeteman, M. Schaap, Q. J. Van den Bouwhuijsen, J. C. Witteman, A. Van der Lugt, L. J. van Vliet et al., "Multispectral MRI centerline tracking in carotid arteries," in SPIE Medical Imaging. International Society for Optics and Photonics, 2011, pp. 79621N-79621N

[204] E. Smistad, T. L. Falch, M. Bozorgi, A. C. Elster, and F. Lindseth, "Medical image segmentation on GPUs-a comprehensive review," Medical Image Analysis, vol. 20, no. 1, pp. 1-18, 2015.

[205] J. Weston, F. Ratle, H. Mobahi, and R. Collobert, "Deep learning via semi-supervised embedding," in Neural Networks: Tricks of the Trade. Springer, 2012, pp. 639-655.

[206] Y. Bengio, "Learning deep architectures for AI," Foundations and Trends in Machine Learning, vol. 2, no. 1, pp. 1-127, 2009.

[207] J. Schmidhuber, "Deep learning in neural networks: An overview," Neural Networks, vol. 61, pp. 85-117, 2015.

[208] J. Donahue, L. Anne Hendricks, S. Guadarrama, M. Rohrbach, S. Venugopalan, K. Saenko, and T. Darrell, "Long-term recurrent convolutional networks for visual recognition and description," in Conference on Computer Vision and Pattern Recognition, 2015, pp. 2625-2634. 